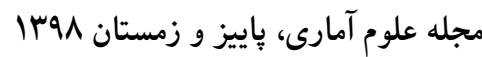

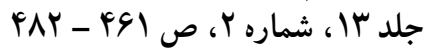

DOI: $10.29252 /$ jss.13.2.461

برآورد بارامترهاى فرآيند يواسون مركب دومتغيرُ دورهاى به روش استنباط حاشيهاى

$$
\text { على سخايى، برويز نصيرى }
$$

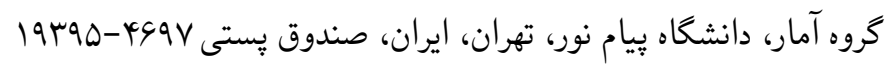

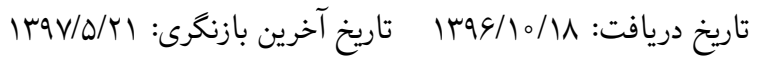

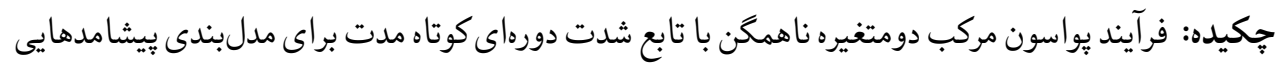

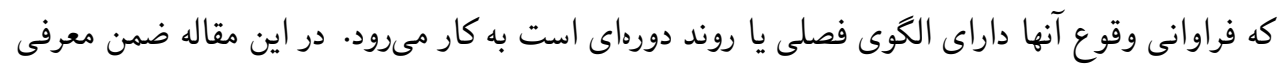

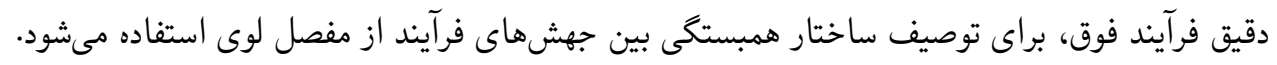

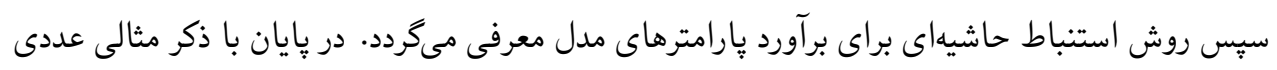

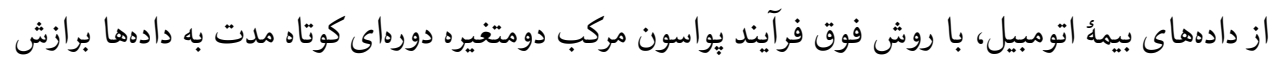

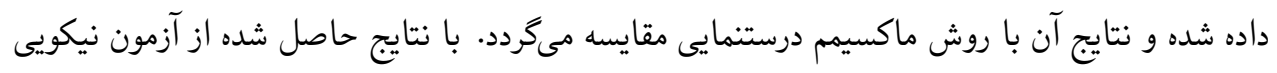

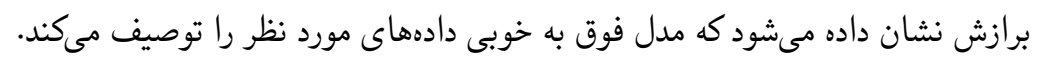

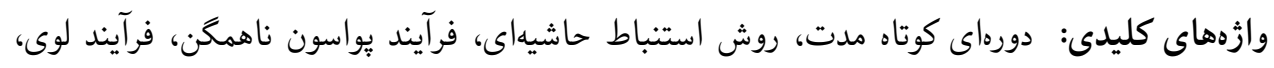

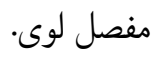

\title{
|
}

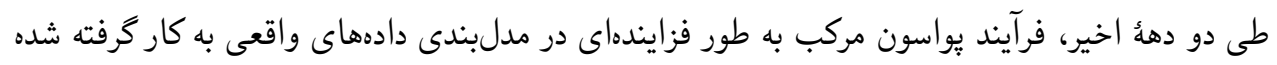

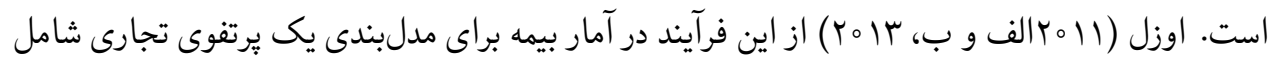

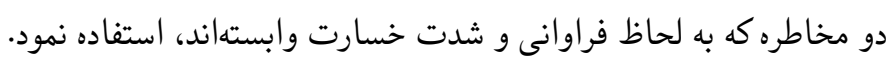

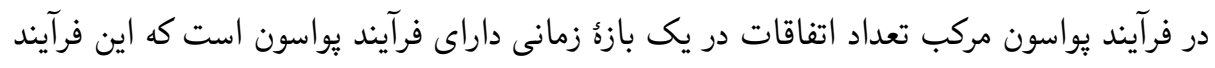

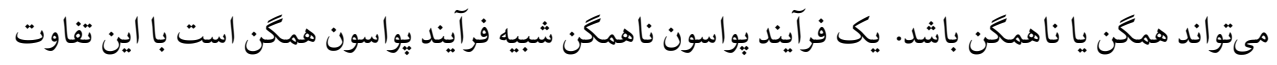


كه نرخ سررسيد اتفاقات با زمان تغيير مىكند. بنابراين، در اين فرآيند نرخ سررسيد در طول دورة مورد بروسى ثابت نيست.

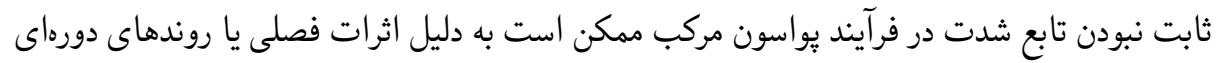

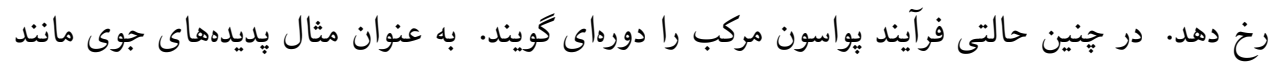

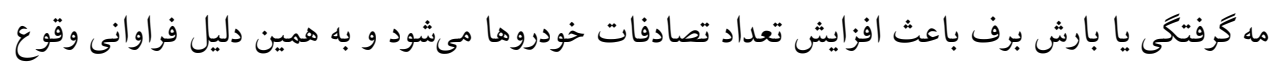

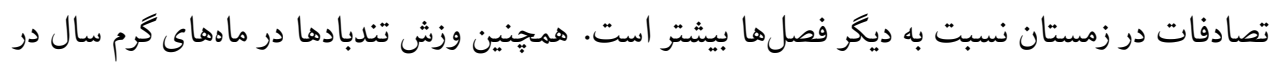

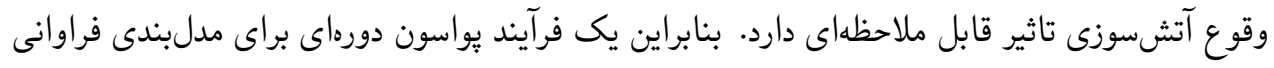

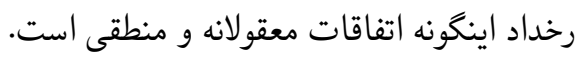

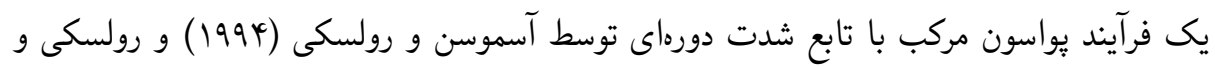

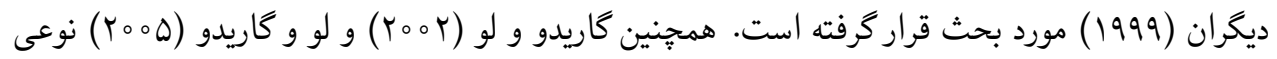

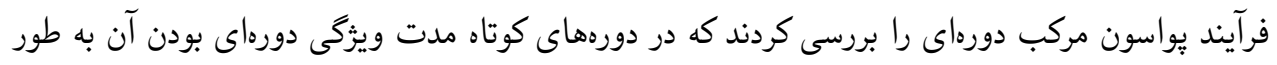

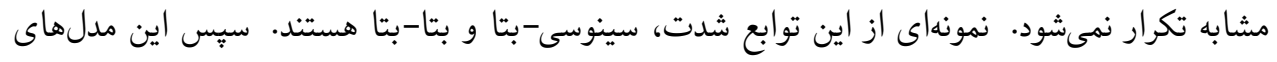

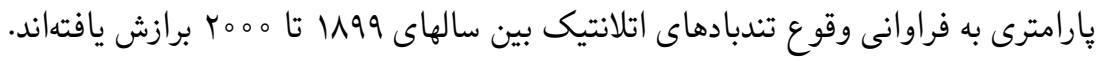

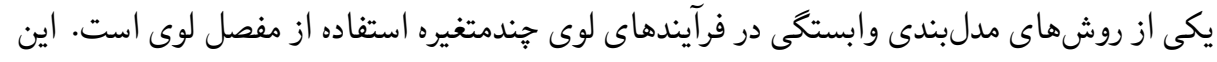

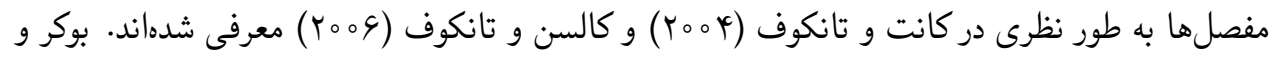

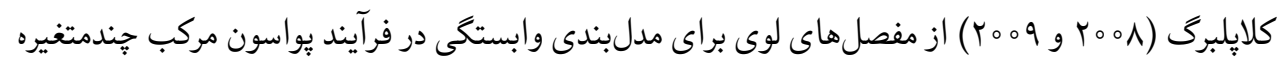

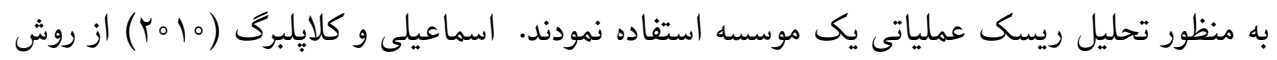

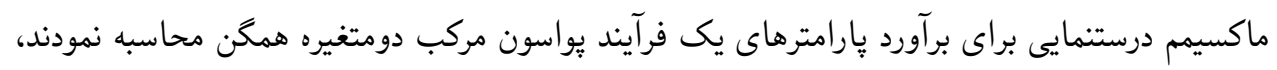

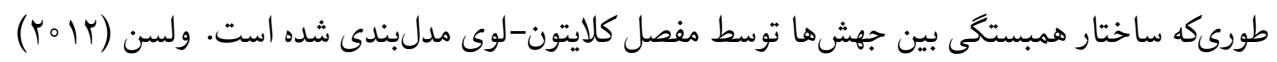

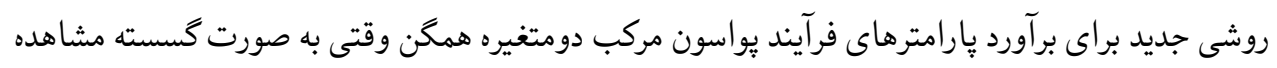
شده باشد، بيشنهاد نمود.

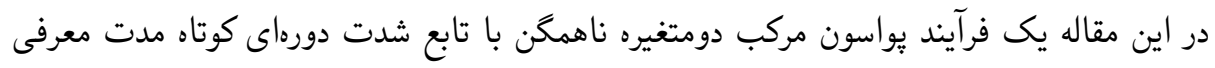

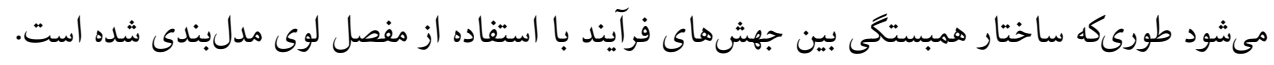

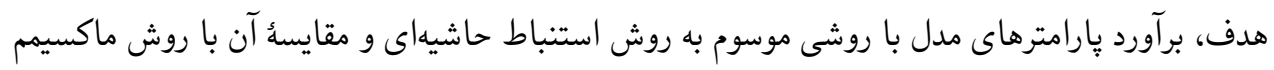


ساختار اين مقاله به صورت زير است: در بخش ب فرآيند بواسون مركب دومتغيره ناهمگن معرفى

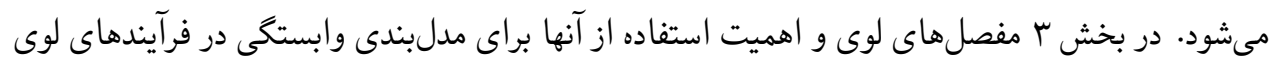

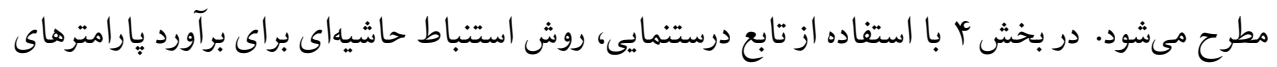

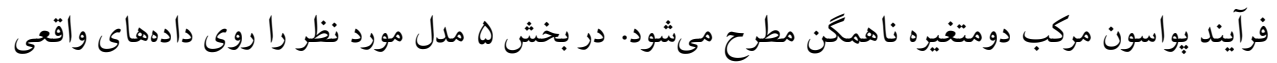

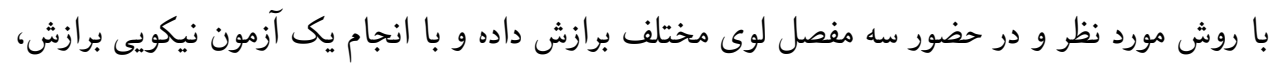
كارايى برازش مدل نيز مورد بررسى قرار مى موديرد.

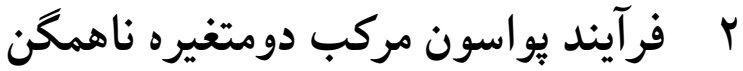

يك فرآيند يواسون مركب دو متغيرهُ به صورت

$$
\boldsymbol{S}(t)=\sum_{i=1}^{N(t)} \boldsymbol{Z}_{i} \quad t \geq \circ
$$

تعريف مىشود، طورى كه مئt

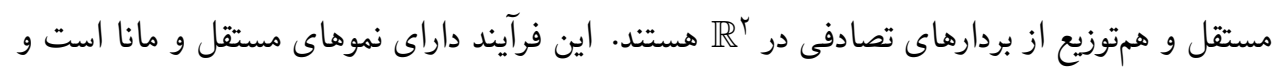

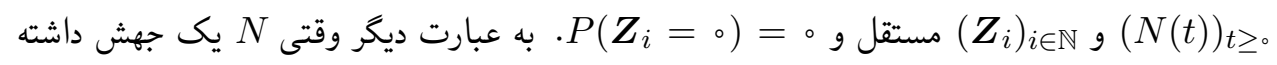
باشد، حداقل يكى از مولفهاى S نيز داراى يك جهش خواهد بود (تقريبا به طور حتم). با استفاده از

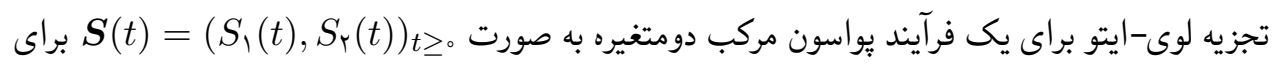
هر

$$
\left(\begin{array}{c}
S_{\uparrow}(t) \\
S_{\curlyvee}(t)
\end{array}\right)=\left(\begin{array}{c}
S_{\perp}^{\perp}(t)+S_{\uparrow}^{\|}(t) \\
S_{\ulcorner}^{\perp}(t)+S_{\uparrow}^{\|}(t)
\end{array}\right) .
$$

فرآيندهاى $(t)$

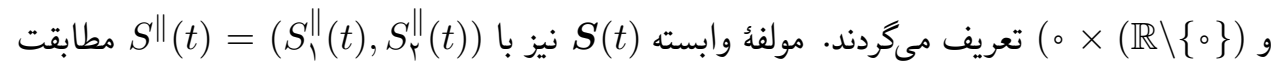

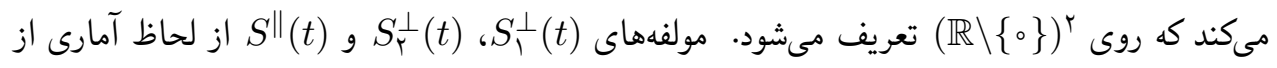

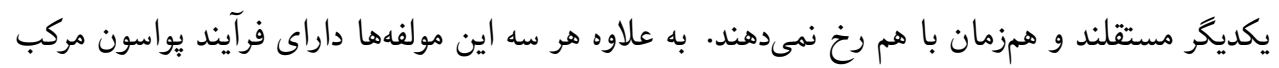


برآورد بارامترهاى فرآيند يواسون مركب دومتغيره ناهمكن

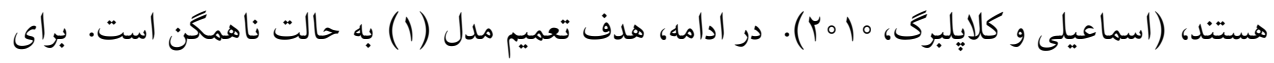

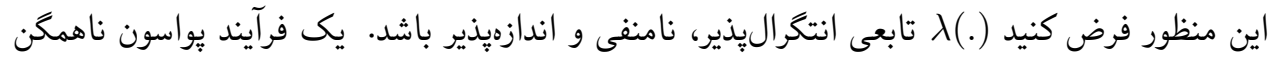
فرئد فرآيند، اكر (T) با بارامتر (T) است، به عبارت ديكر

$$
\begin{aligned}
& P(N(T)=n)=\frac{e^{-\Lambda(T)} \Lambda(T)^{n}}{n !} ; \quad n \in \mathbb{N}, \\
& \text { كه (.) (تابع شدت تجمعى) به صورت }
\end{aligned}
$$

$$
\Lambda(T)=\int_{0}^{T} \lambda(v) d v ; \quad T \geq \bullet,
$$

تعريف مىشود. حالتى را در نظر بكيريد كه تابع شدت فرآيند يواسون ناهمكن تابعى دورهاى با دورة ئه يكسال

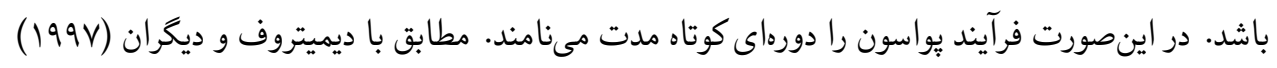

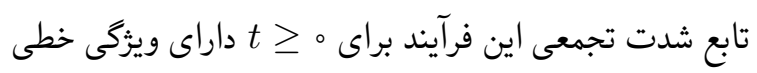

$$
\Lambda(t)=\lfloor t\rfloor \Lambda(1)+\Lambda(t-\lfloor t\rfloor)
$$

است، كه در آن لـ \ـ جزء صحيح t است.

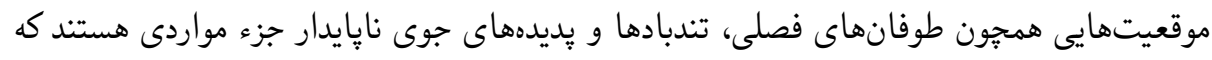

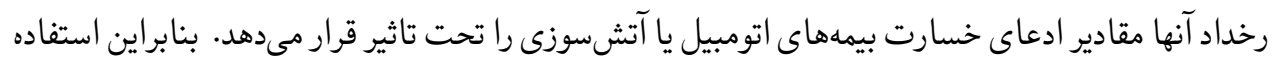

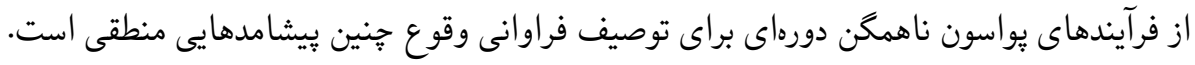

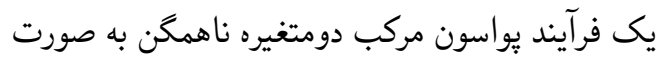

$$
\boldsymbol{S}(t)=\sum_{i=1}^{N(t)}\left(Z_{\uparrow i}, Z_{\curlyvee i}\right)=\left(\sum_{i=\uparrow}^{N(t)} Z_{\uparrow}, \sum_{i=1}^{N(t)} Z_{\curlyvee i}\right) \quad t \geq \bullet,
$$

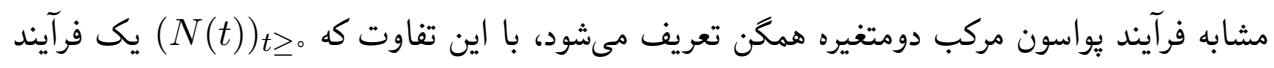

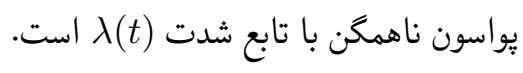




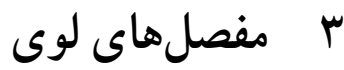

يك فرآيند يواسون مركب دومتغيره نوعى فرآيند لوى است كه براى مدلبندى ساختار همبستگى بين

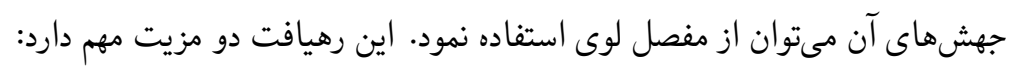

$$
\text { ا ـ. ايجاد مدلى با تعداد پارامترهاى كمتر در مقايسه با حالت معمول. }
$$

r. انعطافيذيرى در ساخت مدلى با تركيب اطلاعات توزيعهاى حاشيهاى و ساختار همبستكى بين

به اين ترتيب در اين روش مدلبندى وابستكى، اخر تابع مفصل معلوم باشد، با مشخص بودن يارامترهاى

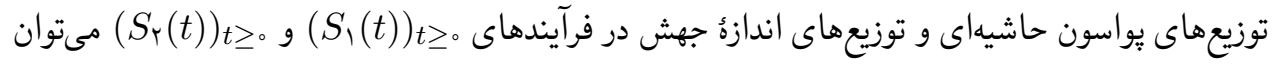
فرآيند يواسون مركب دومتغيره ناهمكن را ايجاد كرد.

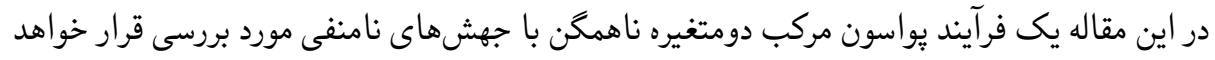

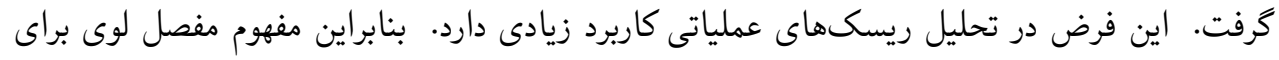
فرآيندهاى بواسون مركب نامنفى ارائه مىشود.

تعريف ا: فرض كنيد П اندازُٔ لوى يك فرآيند لوى دوبعدى با جهشهاى نامنفى باشد. انتكرال دمى اين

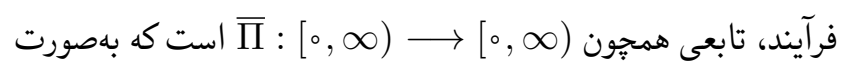

$$
\bar{\Pi}\left(x_{1}, x_{\curlyvee}\right)=\Pi\left(\left[x_{\uparrow}, \infty\right) \times\left[x_{\curlyvee}, \infty\right)\right) \quad\left(x_{1}, x_{\curlyvee}\right) \in[\circ, \infty)^{\Upsilon}
$$

تعريف مىشود و داراى سه ويثگى زير مىباشد: $\bar{\Pi}(\circ, \circ)=\infty \cdot 1$ r. اكر حداقل يكى از آركومانهاى آن مه باشد آنغاه آ برابر صفر است، r.

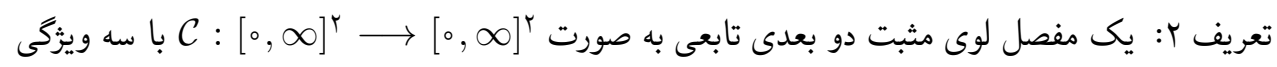
زير است: ا. براى هر كر است $\mathcal{C}\left(u_{1}, u_{r}\right)-\mathcal{C}\left(u_{\uparrow}, v_{r}\right)-\mathcal{C}\left(v_{\uparrow}, u_{\digamma}\right)+\mathcal{C}\left(v_{\uparrow}, v_{r}\right) \geq 。$ 
برآورد پِارامترهاى فرآيند يواسون مركب دومتغيره ناهمكن

$$
\begin{aligned}
& \mathcal{C}\left(u_{1}, \circ\right)=\circ, \mathcal{C}\left(\circ, u_{\digamma}\right)=\circ{ }^{\circ} \mathcal{C}(\circ, \circ)=\circ \cdot r \\
& \text { r }
\end{aligned}
$$

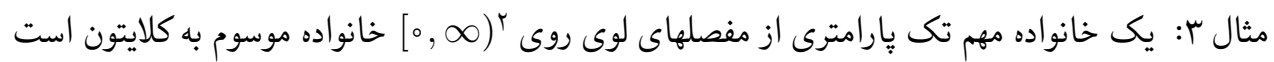

كه بهصورت

$$
\mathcal{C}(u, v ; \alpha)=\left(u^{-\alpha}+v^{-\alpha}\right)^{-\frac{1}{\alpha}} ; \quad u, v>\circ ; \alpha>\circ .
$$

تعريف مىشود. اين مفصل تنها همبستكىهاى مثبت را مدلبندى مىكند. يكى از ويزگكهاى خوب تابع

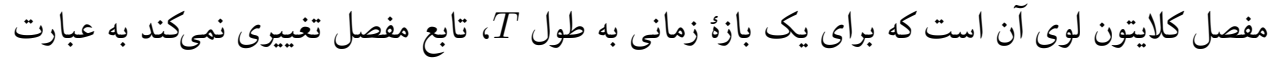
ديغر آنها (دم راست) اتفاق مىافتد.

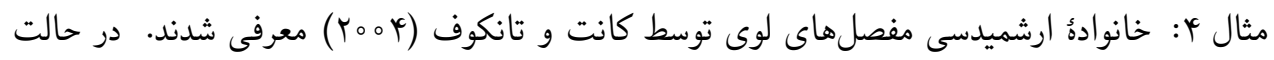

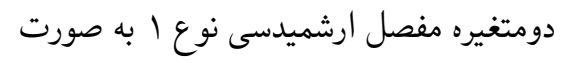

$$
\mathcal{C}(u, v ; \alpha)=\frac{1}{\alpha} \ln \left(\frac{1-e^{-\alpha(u+v)}}{e^{-\alpha u}-r e^{-\alpha(u+v)}+e^{-\alpha v}}\right) ; \quad u, v>\circ ; \alpha>\circ
$$

تعريف مىشود. الكوى وابستكى اين تابع مفصل شبيه مفصل كلايتون لوى است با اين تفاوت كه با $\alpha$ مائ

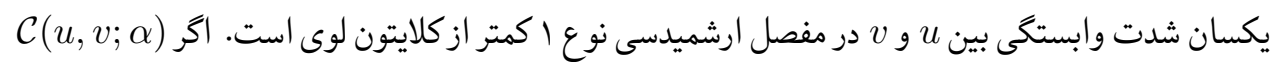

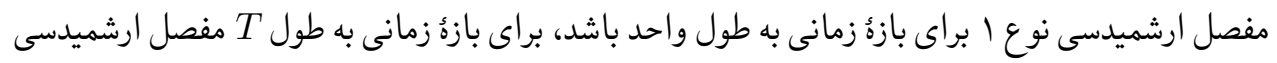
نوع ا مفه مفصل ارشميدسى نوع r در حالت دومتغيره به صورت

$$
\mathcal{C}(u, v ; \alpha)=\ln \left\{\left[\left(e^{u}-1\right)^{-\alpha}+\left(e^{v}-1\right)^{-\alpha}\right]^{-\frac{1}{\alpha}}+1\right\} ; \quad u, v>\circ ; \alpha>\circ,
$$

تعريف مىشود. ساختار وابستكى در اين مفصل از دو مفصل قبلى متمايز است. در واقع اين مفصل علاوه

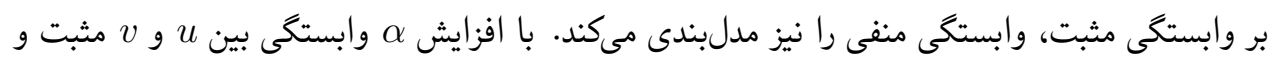

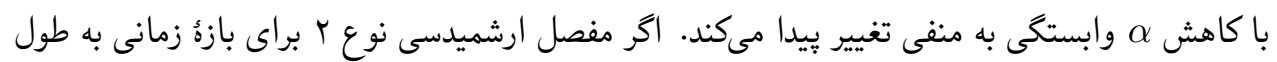

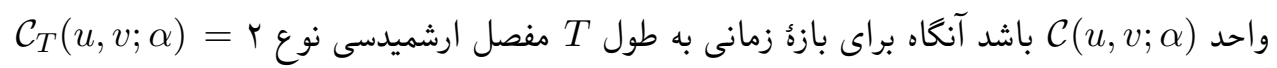




$$
\text { T.C }\left(\frac{u}{T}, \frac{v}{T} ; \alpha\right)
$$

حال به دنبال آن هستيم كه ساختار همبستخى در فرآيند يواسون مركب آن

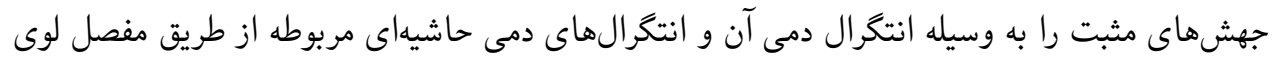

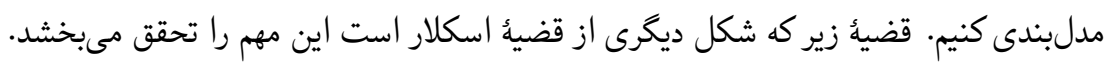

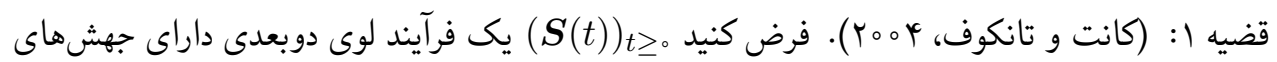
مثبت با انتخرال دمى

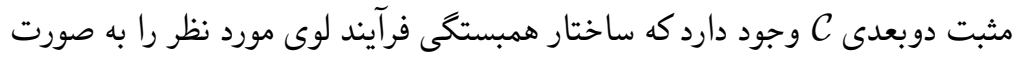

$$
\bar{\Pi}\left(x_{1}, x_{\Upsilon}\right)=\mathcal{C}\left(\bar{\Pi}_{1}\left(x_{1}\right), \bar{\Pi}_{Y}\left(x_{Y}\right) ; \alpha\right) ; \quad x_{1}, x_{\Upsilon} \in[\circ, \infty]
$$

توصيف مىكند. اخر إ و دامندُب

برعكس: فرض كنيد إن

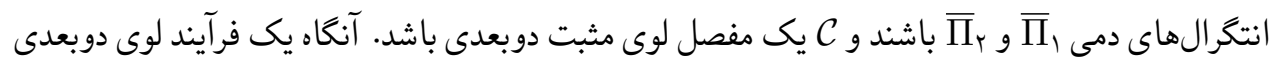

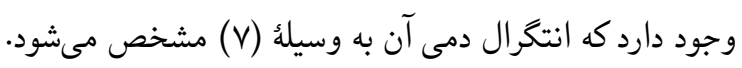
فرض كنيد آ و $\bar{\Pi}$

$$
\begin{aligned}
& \bar{\Pi}_{Y} \text { ب } \bar{\Pi}_{1} \\
& \bar{\Pi}_{i}=\bar{\Pi}_{i}^{\perp}+\bar{\Pi}_{i}^{\|} ; \quad i=1, r,
\end{aligned}
$$

نوشته مىشوند. با استفاده از رابطة اخير مىتوان تابع بقاى اندازءٔ جهشهاى مولفههاى مستقل و وابسته

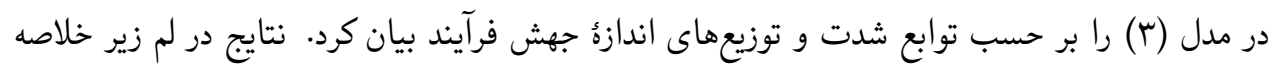
شدهاند.

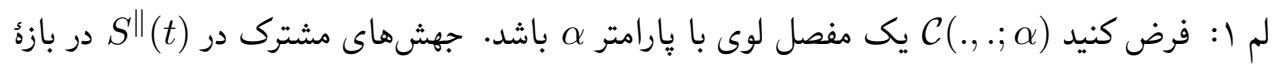
زمانى [ـ

$$
\bar{F}_{T}^{\|}\left(x_{\uparrow}, x_{\curlyvee}\right)=\frac{1}{\Lambda^{\|}(T)} \mathcal{C}\left(\Lambda_{\uparrow}(T) \bar{F}_{\curlywedge}\left(x_{\uparrow}\right), \Lambda_{\curlyvee}(T) \bar{F}_{\Upsilon}\left(x_{\Upsilon}\right) ; \alpha\right) ; \quad x_{\uparrow}, x_{\curlyvee}>\circ .
$$


برآورد پارامترهاى فرآيند يواسون مركب دومتغيره ناهمكن

Y9A

سررسيد مىشوند. به اين ترتيب توابع بقاى حاشيهاى مربوطه عبارتند از:

$$
\begin{aligned}
& \bar{F}_{\backslash T}^{\|}\left(x_{\uparrow}\right)=\frac{1}{\Lambda^{\|}(T)} \mathcal{C}\left(\Lambda_{\uparrow}(T) \bar{F}_{\uparrow}\left(x_{\uparrow}\right), \Lambda_{\uparrow}(T) ; \alpha\right), \\
& \bar{F}_{\Upsilon T}^{\|}\left(x_{\curlyvee}\right)=\frac{1}{\Lambda^{\|}(T)} \mathcal{C}\left(\Lambda_{\uparrow}(T), \Lambda_{\curlyvee}(T) \bar{F}_{\curlyvee}\left(x_{\curlyvee}\right) ; \alpha\right) .
\end{aligned}
$$

جهشهاى مستقل r

$$
\Lambda_{i}^{\perp}(T)=\Lambda_{i}(T)-\Lambda^{\|}(T)
$$

و تابع بقاى اندازهُ جهش

$$
\bar{F}_{i T}^{\perp}(x)=\frac{1}{\Lambda_{i}^{\perp}(T)}\left(\Lambda_{i}(T) \bar{F}_{i}(x)-\Lambda^{\|}(T) \bar{F}_{i T}^{\|}(x)\right)
$$

\section{سررسيد مىشوند.}

\section{F}

يك فرآيند يواسون مركب دومتغيره ناهمكن به صورت مانم

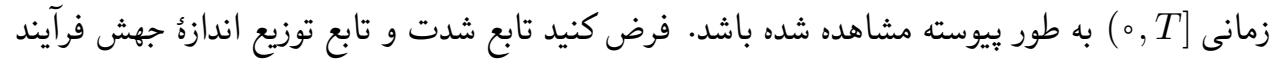
يواسون مركب زمانى

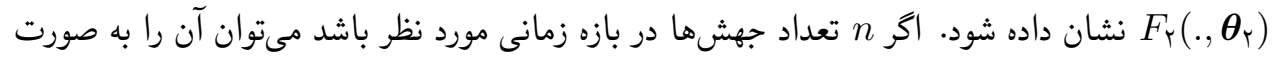

زير تجزيه نمود:

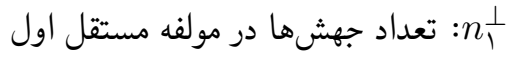

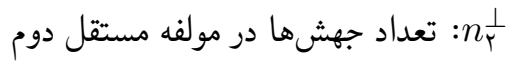

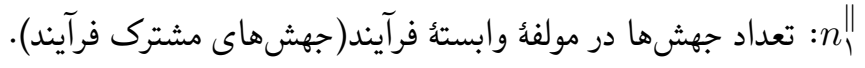

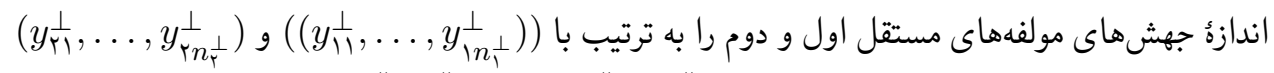

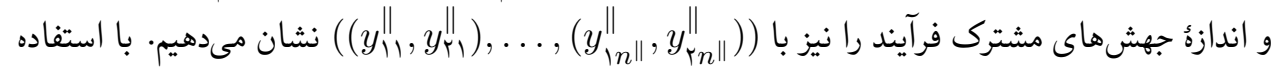




$$
\text { از (Y) تابع درستنمايى را مىتوان به صورت }
$$

$$
\begin{aligned}
& L\left(\alpha, \Lambda_{\uparrow}(T), \Lambda_{\Upsilon}(T), \boldsymbol{\theta}_{\backslash}, \boldsymbol{\theta}_{\Upsilon}\right)=\left(\Lambda_{\uparrow}^{\perp}(T)\right)^{n_{\perp}^{\perp}} e^{-\Lambda_{\uparrow}^{\perp}(T)} \prod_{i=1}^{n_{\perp}^{\perp}}\left[f_{\backslash T}^{\perp}\left(y_{\backslash i}^{\perp} ; \boldsymbol{\theta}_{\uparrow}\right)\right]
\end{aligned}
$$

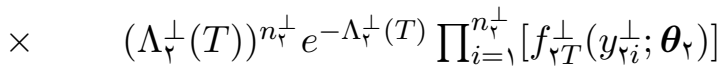

$$
\begin{aligned}
& \times \quad\left(\Lambda^{\|}(T)\right)^{n^{\|}} e^{-\Lambda^{\|}(T)} \prod_{i=1}^{n^{\|}}\left[f_{T}^{\|}\left(\left(y_{\uparrow i}^{\|}, y_{\uparrow i}^{\|}\right) ; \boldsymbol{\theta}_{\uparrow}, \boldsymbol{\theta}_{\Upsilon}\right)\right]
\end{aligned}
$$

$$
\begin{aligned}
& \text { نوشت، كه در آن } \\
& f_{\backslash T}^{\perp}\left(x ; \boldsymbol{\theta}_{\uparrow}\right)=\frac{d}{d x} F_{\backslash T}^{\perp}\left(x ; \boldsymbol{\theta}_{\uparrow}\right)=\frac{\Lambda_{\curlywedge}(T) f_{\uparrow}\left(x ; \boldsymbol{\theta}_{\uparrow}\right)}{\Lambda_{\uparrow}^{\perp}(T)}\left[1-\left.\frac{\partial}{\partial u} \mathcal{C}\left(u, \Lambda_{\uparrow}(T) ; \alpha\right)\right|_{u=\Lambda_{\uparrow}(T) \bar{F}_{\backslash}\left(x ; \boldsymbol{\theta}_{\curlywedge}\right)}\right] \\
& f_{\Upsilon T}^{\perp}\left(y ; \boldsymbol{\theta}_{\Upsilon}\right)=\frac{d}{d y} F_{\Upsilon T}^{\perp}\left(y ; \boldsymbol{\theta}_{\Upsilon}\right)=\frac{\Lambda_{\Upsilon}(T) f_{\Upsilon}\left(y ; \boldsymbol{\theta}_{\Upsilon}\right)}{\Lambda_{\Upsilon}^{\perp}(T)}\left[1-\left.\frac{\partial}{\partial v} \mathcal{C}\left(\Lambda_{\uparrow}(T), v ; \alpha\right)\right|_{v=\Lambda_{\Upsilon}(T) \bar{F}_{\curlyvee}\left(y ; \boldsymbol{\theta}_{\Upsilon}\right)}\right] \\
& f_{T}^{\|}\left(x, y ; \boldsymbol{\theta}_{\uparrow} ; \boldsymbol{\theta}_{\Upsilon}\right)=\frac{\partial^{\Upsilon}}{\partial x \partial y} F_{T}^{\|}\left(x, y ; \boldsymbol{\theta}_{\uparrow} ; \boldsymbol{\theta}_{\Upsilon}\right)=\frac{\Lambda_{\uparrow}(T) \Lambda_{\Upsilon}(T) f_{\uparrow}\left(x ; \boldsymbol{\theta}_{\uparrow}\right) f_{\curlyvee}\left(y ; \boldsymbol{\theta}_{\Upsilon}\right)}{\Lambda^{\|}(T)} \\
& \times \quad\left[\left.\frac{\partial^{r}}{\partial u \partial v} \mathcal{C}(u, v ; \alpha)\right|_{u=\Lambda_{\uparrow}(T) \bar{F}_{\uparrow}\left(x ; \boldsymbol{\theta}_{\uparrow}\right), v=\Lambda_{\curlyvee}(T) \bar{F}_{\curlyvee}\left(y ; \boldsymbol{\theta}_{\curlyvee}\right)}\right] . \\
& \text { با جايكذارى عبارات فوق در (11) لكاريتم تابع درستنمايى به صورت كلى } \\
& l\left(\alpha, \Lambda_{\uparrow}(T), \Lambda_{\uparrow}(T), \boldsymbol{\theta}_{\uparrow}, \boldsymbol{\theta}_{\Upsilon}\right)=n_{\uparrow} \ln \left(\Lambda_{\uparrow}(T)\right)-\Lambda_{\uparrow}(T)+\sum_{i=1}^{n_{\curlywedge}} \ln \left(f_{\uparrow}\left(x_{i} ; \boldsymbol{\theta}_{\uparrow}\right)\right) \\
& +n_{\curlyvee} \ln \left(\Lambda_{\curlyvee}(T)\right)-\Lambda_{\curlyvee}(T)+\sum_{i=1}^{n_{\curlyvee}} \ln \left(f_{\curlyvee}\left(y_{i} ; \boldsymbol{\theta}_{\Upsilon}\right)\right)
\end{aligned}
$$




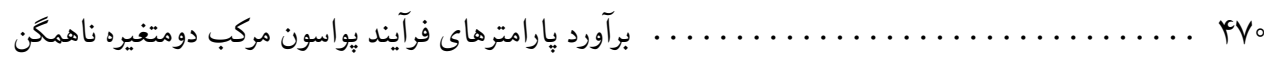

$$
\begin{aligned}
& +\sum_{i=1}^{n_{\perp}^{\perp}} \ln \left(1-\left.\frac{\partial}{\partial u} \mathcal{C}\left(u, \Lambda_{\uparrow}(T) ; \alpha\right)\right|_{u=\Lambda_{\uparrow}(T) \bar{F}_{\backslash}\left(y_{\backslash i}^{\perp} ; \boldsymbol{\theta}_{\uparrow}\right)}\right. \\
& +\sum_{i=1}^{n_{\uparrow}^{\perp}} \ln \left(1-\left.\frac{\partial}{\partial v} \mathcal{C}\left(\Lambda_{\uparrow}(T), v ; \alpha\right)\right|_{v=\Lambda_{\uparrow}(T) \bar{F}_{\curlyvee}\left(y_{\curlyvee i}^{\perp} ; \boldsymbol{\theta}_{\curlyvee}\right)}\right. \\
& +\mathcal{C}\left(\Lambda_{1}(T), \Lambda_{\digamma}(T) ; \alpha\right) \\
& +\sum_{i=1}^{n^{\|}} \ln \left(\left.\frac{\partial^{\Upsilon}}{\partial u \partial v} \mathcal{C}(u, v ; \alpha)\right|_{u=\Lambda_{\uparrow}(T) \bar{F}_{\uparrow}\left(y_{\uparrow i} ; \boldsymbol{\theta}_{\uparrow}\right), v=\Lambda_{\curlyvee}(T) \bar{F}_{\curlyvee}\left(y_{\curlyvee i}^{\|} ; \boldsymbol{\theta}_{\curlyvee}\right)}\right) .
\end{aligned}
$$

بيان مىشود، كه نشان مىدهد تابع درستنمايى مدل (†) را مىتوان به طور كامل برحسب پارامترهاى

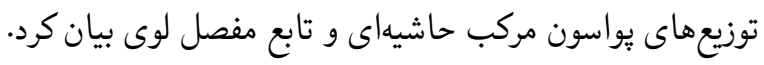

\section{1. روش برآورد استنباط حاشيهاى}

يكى از روشهاى برآورد يارامترهاى رابطُ (r I ) روش يارامترى است. اين روش زمانى به كار مىرود

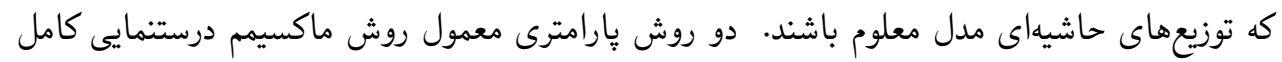

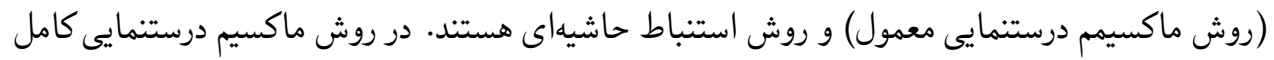

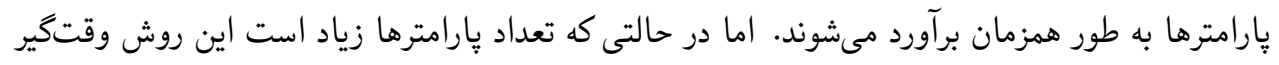

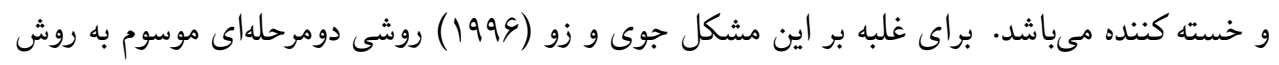

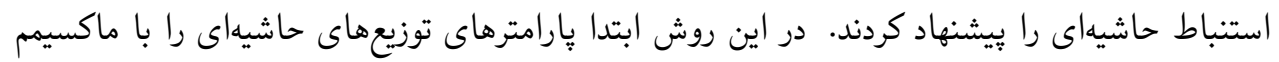
كردن رابطه

$$
\begin{aligned}
& l^{*}\left(\alpha, \Lambda_{\uparrow}(T), \Lambda_{\uparrow}(T), \boldsymbol{\theta}_{\backslash}, \boldsymbol{\theta}_{\uparrow}\right)=n_{\backslash} \ln \left(\Lambda_{\uparrow}(T)\right)-\Lambda_{\backslash}(T)+\sum_{i=1}^{n_{\uparrow}} \ln \left(f_{\uparrow}\left(x_{i} ; \boldsymbol{\theta}_{\uparrow}\right)\right) \\
& +\quad n_{\Upsilon} \ln \left(\Lambda_{\Upsilon}(T)\right)-\Lambda_{\Upsilon}(T)+\sum_{i=1}^{n_{\curlyvee}} \ln \left(f_{\Upsilon}\left(y_{i} ; \boldsymbol{\theta}_{\Upsilon}\right)\right)
\end{aligned}
$$


نسبت به پارامترهاى مورد نظر برآورد نموده سيس با جايگذارى آنها در (Y) (I)، پارامتر(هاى) تابع مفصل

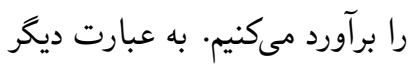

$$
\hat{\alpha}_{I F M}=\underset{\alpha}{\arg \max } l\left(\alpha, \hat{\Lambda}_{1}(T), \hat{\Lambda}_{Y}(T), \hat{\boldsymbol{\theta}}_{,}, \hat{\boldsymbol{\theta}}_{Y}\right)
$$

جدول ا: تعداد جهشهاى مستقل و وابسته در هر ماه براى متغيرهاى ميزان خسارت وارده به اتومبيل

(Y) و جراحتهاى بدنى سرنشينان (X)

\begin{tabular}{|c|c|c|c|c|c|c|c|}
\hline فراواني & $\mathrm{Y}$ & $\mathrm{X}$ & ماه & فراواني & $\mathrm{Y}$ & $\mathrm{X}$ & ماه \\
\hline $\mathrm{V}$ & خسارت & خسارت & \multirow{3}{*}{ ارديبهشت } & 9 & خسارت & خسارت & \multirow{3}{*}{ ل رووردين } \\
\hline v & بدون خسارت & خسارت & & ir & بدون خسارت & خسارت & \\
\hline 1 & خسارت & بدون خسارت & & 1 & خسارت & بدون خسارت & \\
\hline Ir & خسارت & خسارت & \multirow{3}{*}{ تير } & 10 & خسارت & خسارت & \multirow{3}{*}{ خرداد } \\
\hline$\wedge$ & بدون خسارت & خسارت & & 19 & بدون خسارت & خسارت & \\
\hline 1 & خسارت & بدون خسارت & & 。 & خسارت & بدون خسارت & \\
\hline V & خسارت & خسارت & \multirow{3}{*}{ شهريور } & y & خسارت & خسارت & \multirow{3}{*}{ مرداد } \\
\hline 11 & بدون خسارت & خسارت & & 10 & بدون خسارت & خسارت & \\
\hline$r$ & خسارت & بدون خسارت & & r & خسارت & بدون خسارت & \\
\hline$r$ & خسارت & خسارت & \multirow{3}{*}{ آبان } & $y$ & خسارت & خسارت & \multirow{3}{*}{ مهر } \\
\hline r & بدون خسارت & خسارت & & $\wedge$ & بدون خسارت & خسارت & \\
\hline 。 & خسارت & بدون خسارت & & 1 & خسارت & بدون خسارت & \\
\hline 0 & خسارت & خسارت & \multirow{3}{*}{ دى } & $r$ & خسارت & خسارت & \multirow{3}{*}{ آذر } \\
\hline 0 & بدون خسارت & خسارت & & 4 & بدون خسارت & خسارت & \\
\hline 1 & خسارت & بدون خسارت & & 1 & خسارت & بدون خسارت & \\
\hline 9 & خسارت & خسارت & \multirow{3}{*}{ اسفند } & v & خسارت & خسارت & \multirow{3}{*}{ بهمن } \\
\hline V & بدون خسارت & خسارت & & $\wedge$ & بدون خسارت & خسارت & \\
\hline r & خسارت & بدون خسارت & & $r$ & خسارت & بدون خسارت & \\
\hline
\end{tabular}

\section{ه تحليل دادههاى واقعى}

دادهها شامل دو متغير ميزان خسارتهاى وارده به اتومبيل بيمه شدكان (X) و ميزان جراحتهاى بدنى دانى

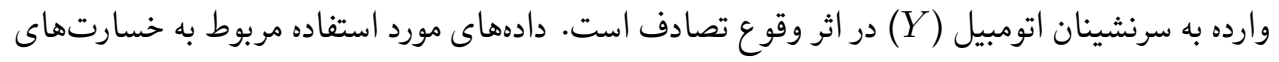


برآورد پارامترهاى فرآيند يواسون مركب دومتغيره ناهمكن

سال هNr| شركت بيمه آسيا هستند كه به صورت ماهيانه كزارش شدهاند. بنا به كفته بيمهكر به علت

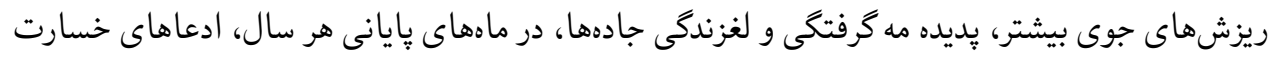

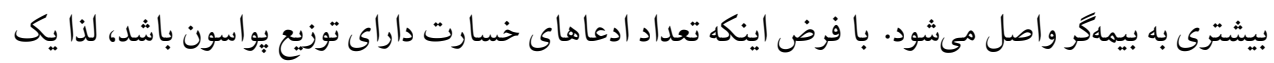

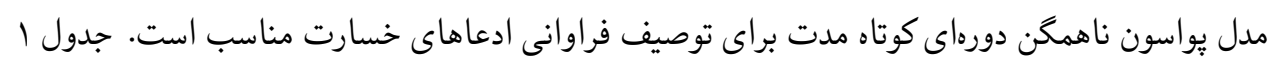

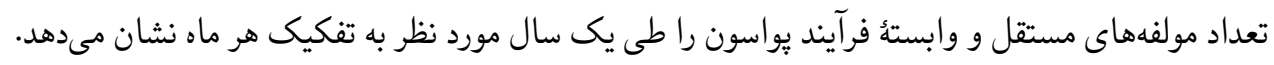

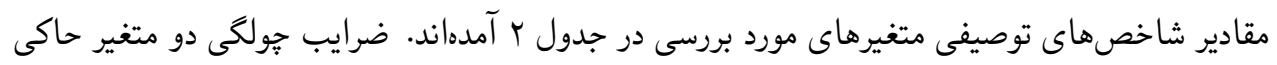
از آن است كه داده ها داراى توزيع دم سنكين نيستند.

جدول ץ: مقادير شاخصهاى توصيفى متغيرهاى خسارت وارده به اتومبيل و جراحتهاى بدنى وارده به سرنشينان آن بدان

\begin{tabular}{|c|c|c|}
\hline جراحتهاى بدنى وارده به سرنشينان & خسارت وارده به اتومبيل & آماره \\
\hline$|\Lambda, T|$ & $\uparrow \wedge, 9 \Lambda$ & ميانكين \\
\hline$r, 49$ & TAl & انحرآف معيار \\
\hline$-0,40$ & $-0,19$ & ضريب جولغّى \\
\hline IT/ND & $r q, \mu$ & كمترين مقدار \\
\hline rT, IV & $\Delta r / 9 \Delta$ & بيشترين مقدار \\
\hline
\end{tabular}

تحليل اكتشافى دادهها با استفاده از آزمون كولموكروف-اسميرنوف نشان مىدهد كه مقادير ادعاهاى

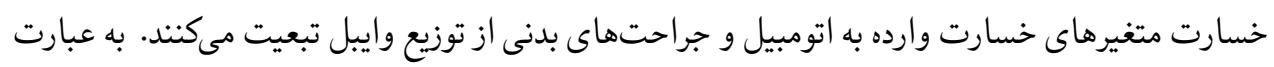
دقيقتر (P)

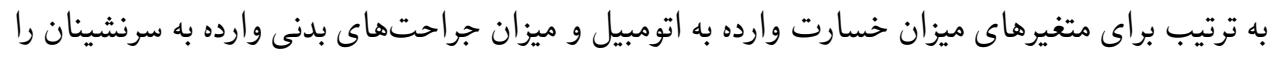

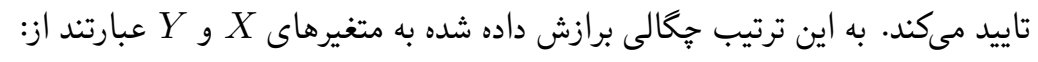

$$
\begin{aligned}
& f_{X}(x)=\frac{r r, r V}{r q, q)}\left(\frac{x}{r q, q)}\right)^{r, r V} \exp \left\{-\left(\frac{x}{r q, q)}\right)^{r r, r v}\right\} ; \quad x>0 \\
& f_{Y}(y)=\frac{9, \Delta \psi}{19, / V}\left(\frac{y}{19, / V}\right)^{\Lambda \Delta \psi} \exp \left\{-\left(\frac{y}{19, / V}\right)^{9, \Delta \psi}\right\} ; \quad y>0 .
\end{aligned}
$$

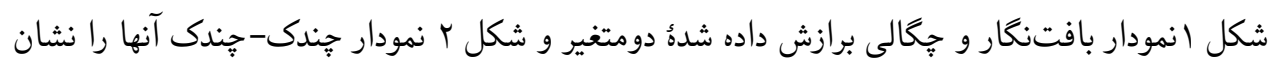


prr على سخايى و برويز نصيرى
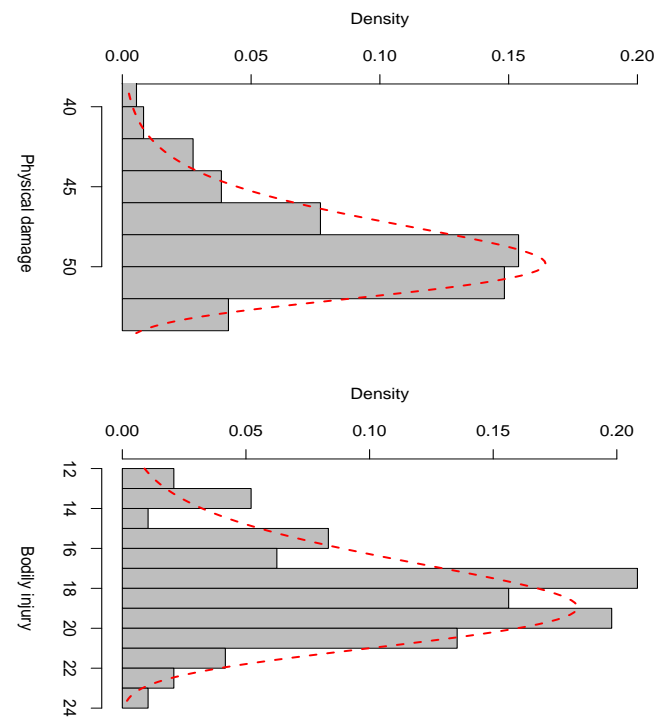

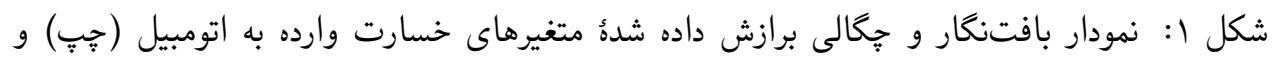
جراحتهاى بدنى (راست).
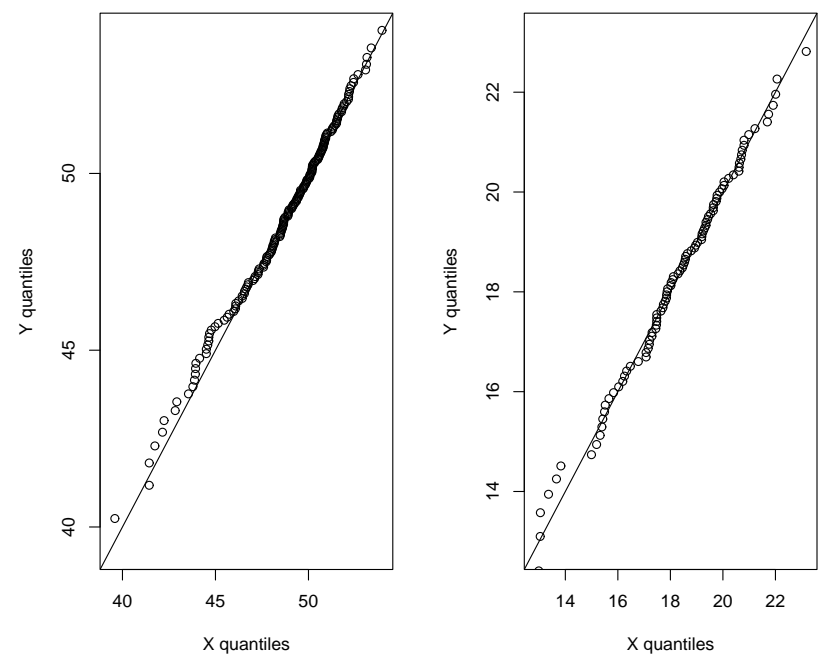

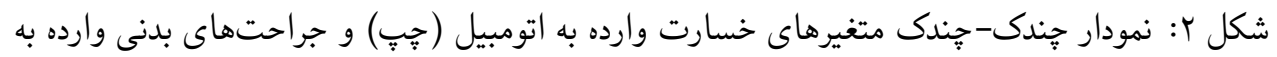
سرنشينان (راست). 
برآورد بارامترهاى فرآيند يواسون مركب دومتغيره ناهمكن

فرض كنيد

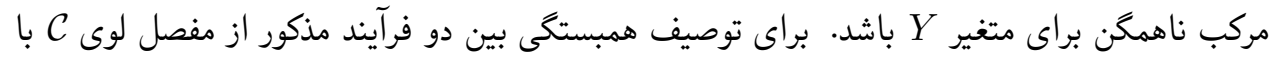

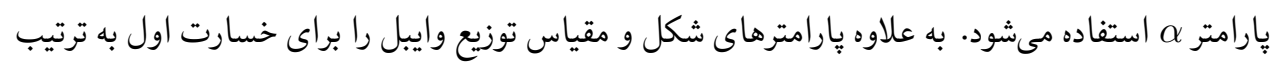

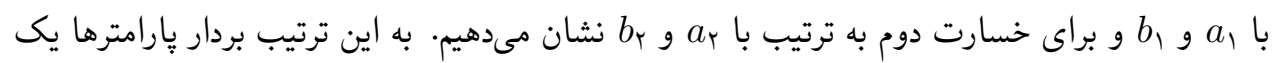

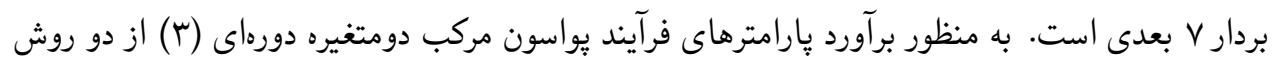

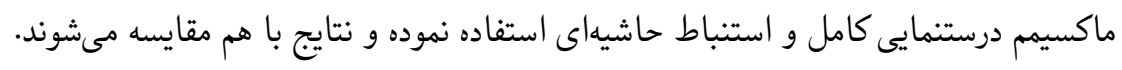

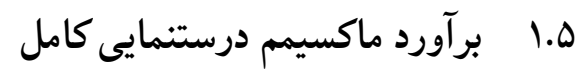

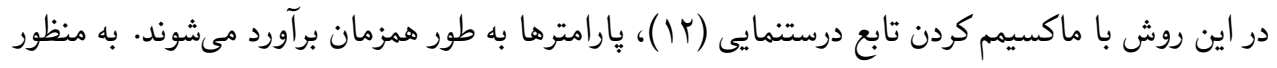

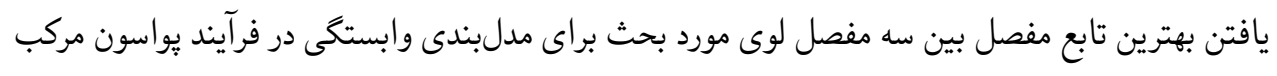

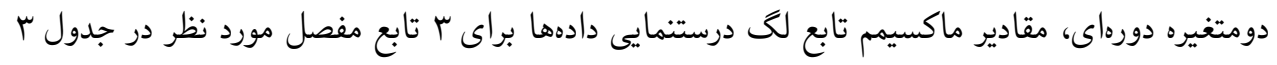

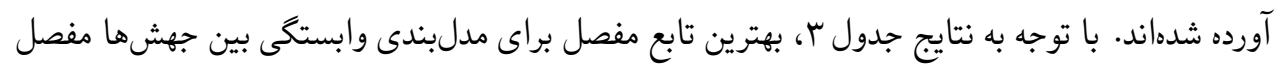

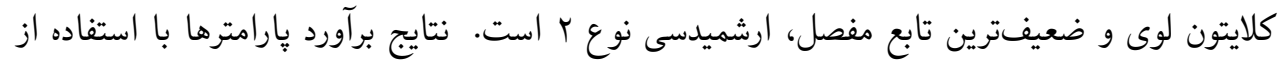

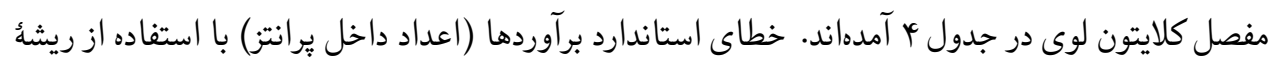
دوم درايه هاى قطر اصلى وارون ماتريس هسين محاسبه شدهاند.

\begin{tabular}{|c|c|c|c|}
\hline \multicolumn{3}{|c|}{ تابع مفصل } & \multirow[b]{2}{*}{ ماه } \\
\hline ارشميدسى نوع r & ارشميدسى نوع | & كلايتون لوى & \\
\hline$-r \Delta, 99^{\circ}$ & $-r r, 009$ & $-r q, \gamma r \Delta$ & فروردين \\
\hline$-44,099$ & $-r_{\Lambda} / 090$ & $-r r / 09 r$ & ارديبششت \\
\hline$-r 9 \wedge \circ 0$ & $-r \Lambda, \Delta<r$ & - & خرداد \\
\hline$-r_{0,091}$ & $-\mid \Lambda, T V G$ & $-\mid T / \Delta \psi_{0}$ & تير \\
\hline - TYMIS & $-9,090$ & oNYK & مرداد \\
\hline$-|M /| V 9$ & 9,949 & $18 / 099$ & شهريور \\
\hline$-\Lambda, \Lambda_{0}$ & $r \Delta, T \Delta Y$ & rTAVE & هـر \\
\hline$-r, 99 \lambda$ & TrSOM & 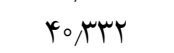 & آبان \\
\hline V,901 & $M, \Lambda \Delta$ & $\Delta 9,400$ & آذر \\
\hline $19,9 \pi T$ & GK, М A & $V 1, \pi 90$ & دى \\
\hline$r, Y, T$ & $\Delta k, y \circ G$ & $99,0 \mathrm{VI}$ & بهمن \\
\hline $10 Y, 0 \Delta r$ & $10 Y, 1 Y 1$ & $111,4 \wedge \mathrm{V}$ & اسفند \\
\hline
\end{tabular}


جدول ع : برآورد بارامترهاى فرآيند بواسون مركب دومتغيره دورهاى به روش ماكسيمم درستنمايى كامل

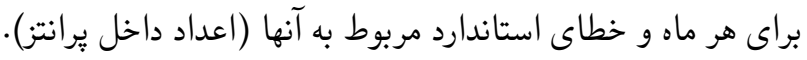

\begin{tabular}{|c|c|c|c|c|c|c|c|}
\hline$b_{r}$ & $a_{r}$ & $b_{1}$ & $a_{1}$ & $\Lambda_{r}(T)$ & $\Lambda_{।}(T)$ & $\alpha$ & پֶارامترها \\
\hline $\begin{array}{l}19, \pi Y 1 \\
(0,901)\end{array}$ & $\begin{array}{l}9 / N Y Y \\
(1, V \Delta G)\end{array}$ & $\begin{array}{l}49 / V Y 1 \\
(0 / 0 \circ 9)\end{array}$ & $\begin{array}{l}T K, T V V \\
(T, K T V)\end{array}$ & $\begin{array}{l}\text { 10,KYY } \\
(r, I V Y)\end{array}$ & $\begin{array}{l}r \backslash, G Y V \\
(Y, g Y \Lambda)\end{array}$ & $\begin{array}{c}r / 94 \lambda \\
(0,991)\end{array}$ & فروردين \\
\hline $\begin{array}{l}19,494 \\
(0,401)\end{array}$ & $\begin{array}{l}10, Y Y Y \\
(1, \Delta Y G)\end{array}$ & $\begin{array}{l}r q, q \uparrow Q \\
(0, \pi \vee q)\end{array}$ & $\begin{array}{l}r H / \wedge \Delta \\
(Y, V \circ \Delta)\end{array}$ & $\begin{array}{l}\text { IV } / \Delta V G \\
(Y, \backslash \Delta Y)\end{array}$ & $\begin{array}{l}r 4,1 \mu_{\Delta} \\
(\Delta, 9 V Y)\end{array}$ & $\begin{array}{c}r / N T \Lambda \\
(0,89 \Lambda)\end{array}$ & ارديبهشت \\
\hline $\begin{array}{l}19 / \wedge \circ 0 \\
(0, \mu \Delta r)\end{array}$ & $\begin{array}{l}10, g \mu^{\circ} \\
(\mid, \pi, I)\end{array}$ & $\begin{array}{l}r q, q \circ 4 \\
(0, \mu \circ 4)\end{array}$ & $\begin{array}{l}Y 1 / \wedge 94 \\
(1 / 90 V)\end{array}$ & $\begin{array}{l}Y V, T \backslash \Lambda \\
(\Delta, I I V)\end{array}$ & $\begin{array}{l}91,9 \wedge \circ \\
(\vee / \wedge \uparrow q)\end{array}$ & $\begin{array}{c}Y, V O Y \\
(0 / \Delta \Delta 1)\end{array}$ & خرداد \\
\hline $\begin{array}{l}19,910 \\
(0, \pi \circ 0)\end{array}$ & $\begin{array}{l}10, \pi, 00 \\
(1,040)\end{array}$ & $\begin{array}{l}49,9 \vee r \\
(0, K q 4)\end{array}$ & $\begin{array}{l}r \mid, M \Lambda_{0} \\
\left(1, \& \mu^{\circ}\right)\end{array}$ & $\begin{array}{l}r q, \mu r \Lambda \\
(9, \lambda I r)\end{array}$ & $\begin{array}{l}1 Y, / q V \\
(q, 0 Y \Delta)\end{array}$ & $\begin{array}{c}r, V \circ V \\
(0, \forall 9 \Lambda)\end{array}$ & تير \\
\hline $\begin{array}{l}19,0 Y 9 \\
(0, \pi V 1)\end{array}$ & $\begin{array}{l}10, \Delta Y \wedge \\
(0,9 V Y)\end{array}$ & $\begin{array}{l}4 q, q 1 Y) \\
(0, K Y V)\end{array}$ & $\begin{array}{l}r \mid, r \wedge q \\
(1,4 \Delta S)\end{array}$ & $\begin{array}{l}\varphi g, 0 \text { r } \\
(\varepsilon, q Y \Delta)\end{array}$ & $\begin{array}{l}99,994 \\
(9 \vee \wedge \wedge r)\end{array}$ & $\begin{array}{c}r, r \Delta \wedge \\
(0, r V T)\end{array}$ & مرداد \\
\hline $\begin{array}{l}19,4 \times 0 \\
(0,494)\end{array}$ & $\begin{array}{c}9,90 \wedge \\
(\circ, / \wedge \circ)\end{array}$ & $\begin{array}{l}r q, q r_{0} \\
\left(0, \pi r_{0}\right)\end{array}$ & $\begin{array}{l}r, /, Y \psi \\
(1, r \wedge r)\end{array}$ & $\begin{array}{l}09,9 \wedge 9 \\
(\vee, 419)\end{array}$ & $\begin{array}{l}110,4 \circ 4 \\
(10,94 \pi)\end{array}$ & $\begin{array}{c}r, 04 q \\
(0, r q 0)\end{array}$ & شهريور \\
\hline $\begin{array}{l}19, \pi \wedge 9 \\
(0, \pi \Delta V)\end{array}$ & $\begin{array}{c}9 / \Delta T I \\
(0 / N \mu \Lambda)\end{array}$ & $\begin{array}{l}\varphi q, \wedge q \vee \\
(0, Y \circ \wedge)\end{array}$ & 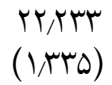 & $\begin{array}{l}9 Y, Y q 9 \\
(V, V V G)\end{array}$ & $\begin{array}{l}|T V, \mathscr{G}| \\
(11, K \Psi Y)\end{array}$ & $\begin{array}{c}1,994 \\
(0, \pi 99)\end{array}$ & مهر \\
\hline $\begin{array}{l}19, \pi \Delta T \\
(0, \pi \Delta 1)\end{array}$ & $\begin{array}{c}9 / 0 T \Lambda \\
(0, N \backslash \Lambda)\end{array}$ & $\begin{array}{l}r q, \wedge \xi_{0} \\
\left(0, r_{\circ}<\right)\end{array}$ & $\begin{array}{l}r 1,990 \\
\left(1, / 99^{\circ}\right)\end{array}$ & $\begin{array}{l}90 / 419 \\
(V / 901)\end{array}$ & $\begin{array}{l}1 \% 4, \times 91 \\
(11,000)\end{array}$ & $\begin{array}{l}1,919 \\
(0,591)\end{array}$ & آبان \\
\hline $\begin{array}{l}19, \pi 9 V \\
(0, \pi r V)\end{array}$ & $\begin{array}{c}9 / \wedge \circ \Lambda \\
(\circ, N Y Y)\end{array}$ & $\begin{array}{l}r q, \wedge k Y \\
(0, / 9 V)\end{array}$ & $\begin{array}{l}Y Y, \circ Y Y \\
(I, T O Y)\end{array}$ & $\begin{array}{l}9 q, 04 q \\
(\Lambda, \Delta Q Y)\end{array}$ & $\begin{array}{l}\mid K F, Y \wedge D \\
(11, \wedge V Y)\end{array}$ & $\begin{array}{c}1,991 \\
(0, Y \Delta Y)\end{array}$ & آذر \\
\hline $\begin{array}{l}19, \pi 00 \\
(0, \pi Y V)\end{array}$ & $\begin{array}{c}9 / V 14 \\
(0,994)\end{array}$ & $\begin{array}{l}4 q, \wedge \wedge 0 \\
(0, / \wedge 9)\end{array}$ & $\begin{array}{l}K Y, D \backslash \Lambda \\
\left(I, K Y_{0}\right)\end{array}$ & $\begin{array}{l}V Y, F \Lambda Y \\
(\Lambda, F \vee q)\end{array}$ & $\begin{array}{l}1 \Delta K \Delta V Y T \\
(1 K, T G \Lambda)\end{array}$ & $\begin{array}{c}1,9 H 4 \\
(0, K r V)\end{array}$ & دى \\
\hline $\begin{array}{l}19, \pi 90 \\
(0, \pi, \pi)\end{array}$ & $\begin{array}{c}9 / V V V^{c} \\
(0,9 \Delta \Delta)\end{array}$ & $\begin{array}{l}\varphi q, \wedge q \mu \\
(0, / \vee q)\end{array}$ & $\begin{array}{l}r Y, N Y q \\
(1, \wedge q)\end{array}$ & $\begin{array}{l}\Lambda 0 / \wedge \circ \mu \\
(9,190)\end{array}$ & $\begin{array}{l}199,9400 \\
(1 K, 940)\end{array}$ & $\begin{array}{c}1,909 \\
(0, K Y 0)\end{array}$ & بهمن \\
\hline $\begin{array}{l}19,1 \circ 9 \\
(0, \pi \circ 9)\end{array}$ & $\begin{array}{l}9, Y V Y \\
(0,900)\end{array}$ & $\begin{array}{l}49,140 \\
(0,199)\end{array}$ & $\begin{array}{l}r Y, q V_{0} \\
(1 /, Q Y)\end{array}$ & $\begin{array}{l}90, q Y \wedge \\
(9, q M Y)\end{array}$ & $\begin{array}{l}\text { INOAMA } \\
(I K \Delta \circ K)\end{array}$ & $\begin{array}{c}1 /, 49 \\
(0, \pi \circ 1)\end{array}$ & اسفند \\
\hline
\end{tabular}

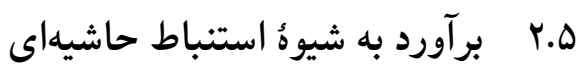

در روش برآورد استنباط حاشيهاى برآورد پارامترهاى توزيعهاى حاشيهاى فرآيند يواسون مركب ناهمخن

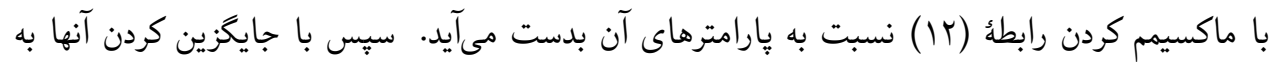

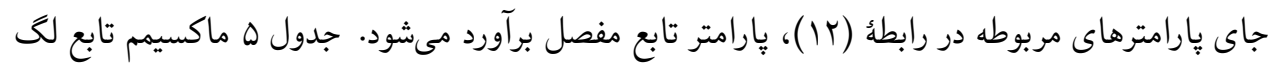

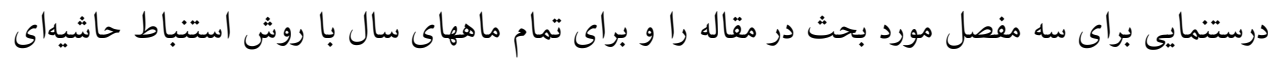


برآورد پارامترهاى فرآيند يواسون مركب دومتغيره ناهمكن

\begin{tabular}{|c|c|c|c|}
\hline \multicolumn{3}{|c|}{ تابع مفصل } & \multirow[b]{2}{*}{ ماه } \\
\hline ارشميدسى نوع r & ارشميدسى نوع ا & كلايتون لوى & \\
\hline$-4 G / 011$ & $-r 4,919$ & $-r \circ \Delta R T$ & فروردين \\
\hline$-r \Delta, 0 \mid \Lambda$ & $-r q, \pi \Delta q$ & $-r 0, r 99$ & ارديبهشت \\
\hline$-r q, 1<q$ & $-r q / \wedge \wedge \Delta$ & - & خرداد \\
\hline - MGNMT & $-19 / 190$ & $-I T, Y V A$ & تير \\
\hline - & $-v / 909$ & orNAr & مرداد \\
\hline$-r \Lambda, V \nu$ & V/DGY & $19 \pi / r$ & شهريور \\
\hline$-r \Delta, \circ p r$ & TY/OTY & $M T / \| F$ & مهر \\
\hline$-M T / \Delta \Delta_{0}$ & rq,V90 & ו וسזרם & آبِان \\
\hline$-M T / D Y Y$ & $40 / 1 \circ 9$ & $\Delta \Delta, \pi \Delta V$ & آذر \\
\hline$-\mid \Lambda_{1} / \varphi_{0}$ & 09,919 & $V 0, K \varepsilon \Lambda$ & دى \\
\hline$-15,4 r q$ & ATNGV & 94,910 & بهمن \\
\hline$-10,109$ & $91,9 \wedge 9$ & $110 / \mathrm{MV}$ & اسفند \\
\hline
\end{tabular}

همانتد روش ماكسيمم درستنمايى كامل، مفصل كلايتون لوى، بهترين تابع مفصل و مفصل ارشميدسى

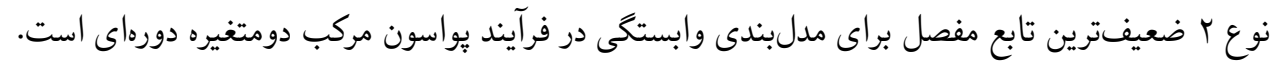

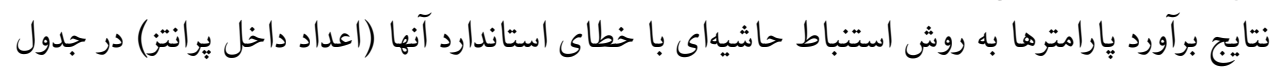
و آمده است.

بررسى دو جدول Y و و نشان مىدهد كه با افزايش طول بازُٔ زمانى مورد نظر (افزايش ماههاى مورد

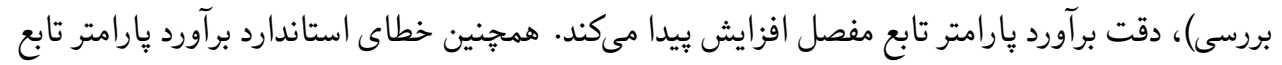

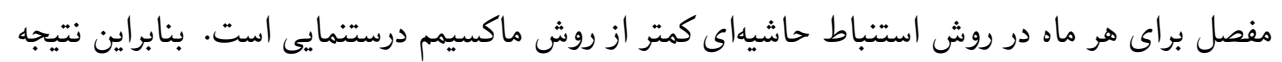
مىشود روش استنباط حاشيهاى دقت بالاترى دارد.

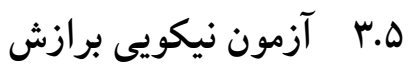

به منظور ارزيابى نيكويى برازش وابستكى بين حاشيهها با استفاده از مفصل كلايتون لوى در فرآيند يواسون

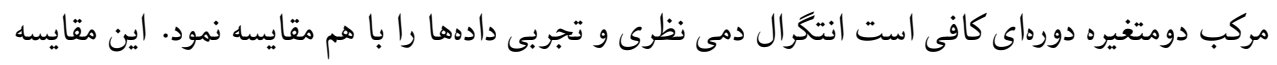

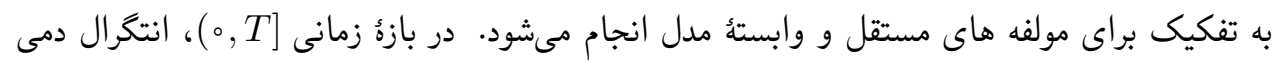


جدول 9: برآورد بارامترهاى فرآيند يواسون مركب دومتغيره ناهمخن به روش استنباط حاشيهاى براى هر

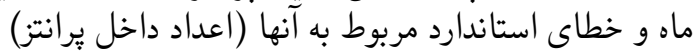

\begin{tabular}{|c|c|c|c|c|c|c|c|}
\hline$b_{Y}$ & $a_{\curlyvee}$ & $b_{1}$ & $a_{1}$ & $\Lambda_{\curlyvee}(T)$ & $\Lambda_{1}(T)$ & $\alpha$ & پارامترها \\
\hline $\begin{array}{l}19,400 \\
(0,9 Y Y)\end{array}$ & $\begin{array}{l}\text { lo, FOY } \\
\text { (Y,OYYT) }\end{array}$ & $\begin{array}{l}r q, q v r \\
\left(0 / \Delta V^{k}\right)\end{array}$ & $\begin{array}{l}\text { 19/AVD } \\
\text { (r/KYV }\end{array}$ & $\begin{array}{c}10 \\
(Y / \mid G Y)\end{array}$ & $\begin{array}{c}r \mid \\
(r, \Delta \wedge r)\end{array}$ & $\begin{array}{c}\text { Y,AGY } \\
(0, N T \Delta)\end{array}$ & فروردين \\
\hline $\begin{array}{l}\text { 19,DYV } \\
(0, K Y Y)\end{array}$ & $\begin{array}{l}11, \Delta q^{\prime} \\
(Y, T Y Y)\end{array}$ & $\begin{array}{l}49,9 \% 0 \\
(0,4 r \Delta)\end{array}$ & $\begin{array}{l}r \circ, G \wedge r \\
\left(r \wedge V_{0}\right)\end{array}$ & $\begin{array}{c}11 \\
(k, N \in Y)\end{array}$ & $\begin{array}{c}\text { ro } \\
(\Delta, 9 \mid 9)\end{array}$ & $\begin{array}{l}r, \Delta \Delta Q \\
(0,4 Q Y)\end{array}$ & ارديبهشت \\
\hline $\begin{array}{l}19, N V K \\
(0, M K Y)\end{array}$ & $\begin{array}{l}11,9 \circ 1 \\
(1, N<4)\end{array}$ & $\begin{array}{l}r q, M \Lambda 1 \\
(0, \mu Y Y)\end{array}$ & $\begin{array}{l}Y O N T Y Y \\
(Y, \backslash \Delta Y)\end{array}$ & $\begin{array}{c}r \Lambda \\
(\Delta, K q Y)\end{array}$ & $\begin{array}{c}91 \\
(\mathrm{~V} / 10)\end{array}$ & $\begin{array}{c}r, 949 \\
(0, \pi 90)\end{array}$ & خرداد \\
\hline $\begin{array}{l}19, \Delta \varphi \Delta \\
(0, \mu \circ \varphi)\end{array}$ & $\begin{array}{l}10 / \Delta K \psi \\
(1, / \wedge 0)\end{array}$ & $\begin{array}{l}0,00 \mathrm{~V} \\
(0, \pi 94)\end{array}$ & $\begin{array}{l}\text { TI/9YG } \\
(1 / 9 r \Delta)\end{array}$ & $\begin{array}{c}\psi \mid \\
(\varepsilon, \psi \circ r)\end{array}$ & $\begin{array}{c}11 \\
(9,000)\end{array}$ & $\begin{array}{c}r, 991 \\
(0, \pi T Y)\end{array}$ & تير \\
\hline $\begin{array}{l}19, \Delta \vee G \\
(0, T \vee \Delta)\end{array}$ & $\begin{array}{l}10,940 \\
(1, \pi+\Lambda)\end{array}$ & $\begin{array}{l}4 q, q \Delta V \\
(0, r \Delta \Delta)\end{array}$ & $\begin{array}{l}Y \backslash /, \circ D \\
(I, N Y Y)\end{array}$ & $\begin{array}{c}Y V \\
(\varphi, \wedge \Delta \varphi)\end{array}$ & $\begin{array}{c}90 \\
\left(9 / V^{*} V\right)\end{array}$ & $\begin{array}{l}r, T V G \\
(0, T V I)\end{array}$ & مرداد \\
\hline $\begin{array}{l}19,0 H T \\
(0, K 94)\end{array}$ & $\begin{array}{l}10, \mu \circ 0 \\
(1,0 Y V)\end{array}$ & $\begin{array}{l}r q, q 4 q \\
(0, K H Y)\end{array}$ & $\begin{array}{l}r \mid, \mu K Y \\
(1, \Delta \Lambda \Lambda)\end{array}$ & $\begin{array}{c}\Delta V \\
(V / \Delta \Delta \circ)\end{array}$ & $\begin{array}{c}11 \% \\
\left(10, g \mu_{0}\right)\end{array}$ & $\begin{array}{c}r / 091 \\
(0, T Y 0)\end{array}$ & شهريور \\
\hline $\begin{array}{l}19,497 \\
(0, \pi 90)\end{array}$ & $\begin{array}{l}10,099 \\
(0,999)\end{array}$ & $\begin{array}{l}4 q, q 4 r \\
(0, \pi / 9)\end{array}$ & $\begin{array}{l}r 1 /, 199 \\
(1 / 019)\end{array}$ & $\begin{array}{c}G Y \\
(V \wedge Y Y)\end{array}$ & $\begin{array}{c}11 \mathrm{ro}^{2} \\
\left(11 / \lambda \Lambda_{0}\right)\end{array}$ & $\begin{array}{l}r, \circ r \Lambda \\
(0, r \circ \Delta)\end{array}$ & مهر \\
\hline $\begin{array}{l}\mid 9,4 \times 1 \\
(0, \pi \Delta 1)\end{array}$ & $\begin{array}{l}10,190^{\circ} \\
(0,90 r)\end{array}$ & $\begin{array}{l}r q, \wedge V V^{f} \\
(0, \pi \mid q)\end{array}$ & $\begin{array}{l}r, / K Y K \\
(1,4 \Delta \Lambda)\end{array}$ & $\begin{array}{c}90 \\
(\Lambda, 04)\end{array}$ & $\begin{array}{c}1 \% r \\
(11,4 \wedge 9)\end{array}$ & $\begin{array}{l}r, 0 Y G \\
(0,19 \Lambda)\end{array}$ & آبان \\
\hline $\begin{array}{l}19,011 \\
(0, \pi T V)\end{array}$ & $\begin{array}{l}10,490 \\
(0,90 Y)\end{array}$ & $\begin{array}{l}r q, \wedge \Delta q \\
(\circ, Y \circ q)\end{array}$ & $\begin{array}{l}r \mid / \Delta 01 \\
(|,| Y \mid r)\end{array}$ & $\begin{array}{c}99 \\
(\Lambda, \mu \vee \nu)\end{array}$ & $\begin{array}{c}|Y| \\
(11 / \wedge V k)\end{array}$ & $\begin{array}{l}1,991 \\
(0,190)\end{array}$ & آذر \\
\hline $\begin{array}{l}19,449 \\
(0, \pi+1)\end{array}$ & $\begin{array}{l}|0,4| V \\
(0,910)\end{array}$ & $\begin{array}{l}49,191 \\
(0,199)\end{array}$ & $\begin{array}{l}r \mid, N r G \\
(1, Y \circ r)\end{array}$ & $\begin{array}{c}V \Delta \\
\left(\Lambda, 99_{0}^{\circ}\right)\end{array}$ & $\begin{array}{c}101 \\
(1 K, K M A)\end{array}$ & $\begin{array}{l}1,991 \\
(0,179)\end{array}$ & دى \\
\hline $\begin{array}{l}19, \pi 94 \\
(0, \pi / 0)\end{array}$ & $\begin{array}{l}10, \pi Y V \\
(0, N 01)\end{array}$ & $\begin{array}{l}4 q, \wedge \vee q \\
(0, \wedge \wedge \varphi)\end{array}$ & $\begin{array}{l}r|, N| \Lambda \\
(1, \pi+4)\end{array}$ & $\begin{array}{c}10 \\
(q, Y Y \circ)\end{array}$ & $\begin{array}{c}199 \\
(1 K / M Y)\end{array}$ & $\begin{array}{c}1,949 \\
(0,19 V)\end{array}$ & بهمن \\
\hline $\begin{array}{l}19,1 V T \\
(0, \pi 19)\end{array}$ & $\begin{array}{c}q / \Delta r q \\
(0, v \Delta r)\end{array}$ & $\begin{array}{l}4 q, q \circ q \\
(0, / \vee 0)\end{array}$ & $\begin{array}{l}r r, r G \Lambda \\
(1, \pi \circ 9)\end{array}$ & $\begin{array}{c}99 \\
(9 \times 91)\end{array}$ & $\begin{array}{c}\text { MKr } \\
(|r, \times 9|)\end{array}$ & $\begin{array}{l}1 / \wedge 94 \\
(0, / \Delta 4)\end{array}$ & اسفند \\
\hline
\end{tabular}

$$
\begin{aligned}
& \text { تجربى مولفهاى مستقل به صورت }
\end{aligned}
$$

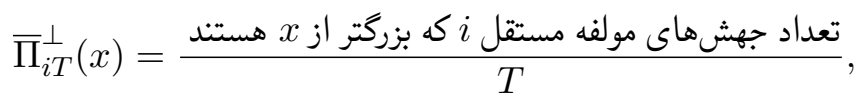

$$
\begin{aligned}
& \text { و انتخرال دمى تجربى مولفههاى وابسته نيز به صورت } \\
& \bar{\Pi}_{i T}^{\|}(x)=\frac{\text { تعداد جهشهاى وابسته در مولفه } i \text { كه بزركتر از x هستند }}{T}
\end{aligned}
$$

تعريف مى شوند. انتخرال دمى نظرى مولفهُ وابستهُ اول، مولفهُ وابستُ دوم و مولفهاى مستقل در بازهُ 
ب. برآورد بارامترهاى فرآيند بواسون مركب دومتغيره ناهمكن

زمانى [o, ) مطابق تعاريف و مفاهيم بخش ب به ترتيب عبارتند از:

$$
\begin{aligned}
& \bar{\Pi}_{\backslash T}^{\|}(x)=\mathcal{C}\left(\Lambda_{\uparrow}(T), \bar{F}_{\uparrow}(x), \Lambda_{\curlyvee}(T) ; \alpha\right), \\
& \bar{\Pi}_{\Upsilon T}^{\|}(x)=\mathcal{C}\left(\Lambda_{\uparrow}(T), \Lambda_{\mathrm{r}}(T), \bar{F}_{\mathrm{r}}(x) ; \alpha\right), \\
& \bar{\Pi}_{i T}^{\perp}(x)=\Lambda_{i}(T) \bar{F}_{i}(x)-\bar{\Pi}_{i T}^{\|}(x) \quad ; \quad i=\text {, }, \text { ケ. }
\end{aligned}
$$

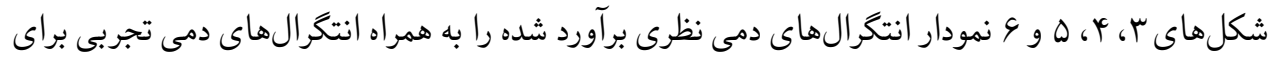

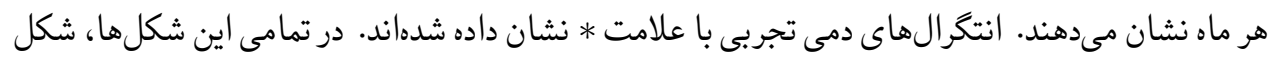

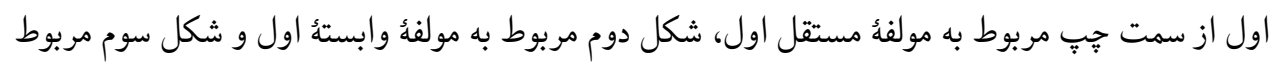

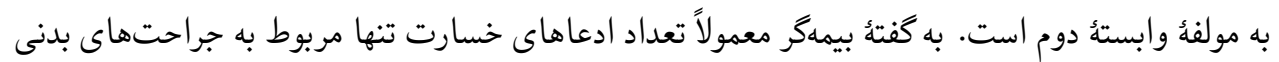

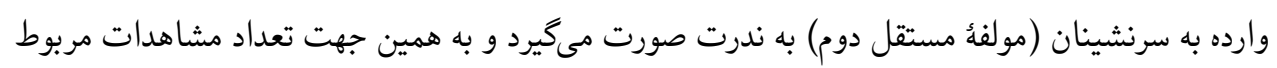

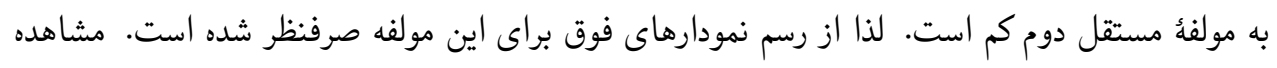

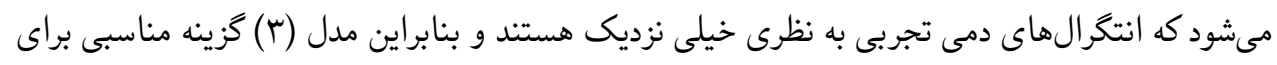
توصيف دادههاى واقعى است.

\section{بحث و نتيجهگيرى}

در اين مقاله نوعى فرآيند يواسون مركب دومتغيره ناهمكن معرفى شد، كه تعداد جهشهاى آن آن از فرآيند

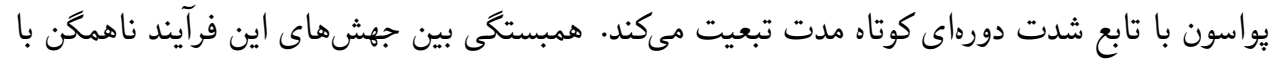

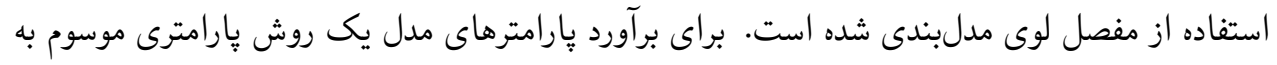

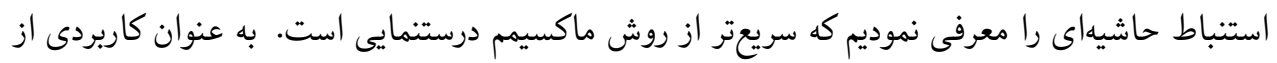

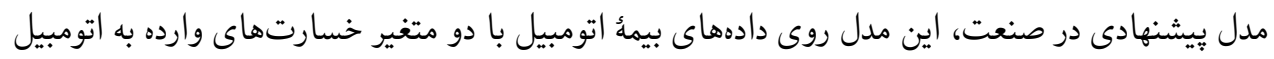

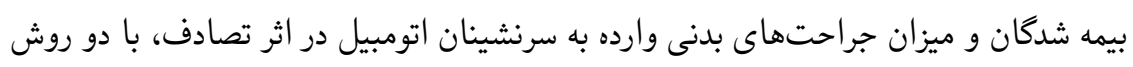

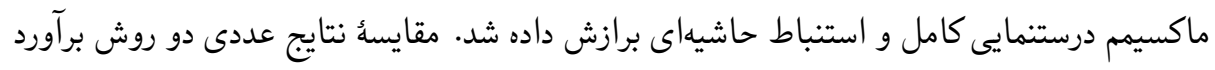

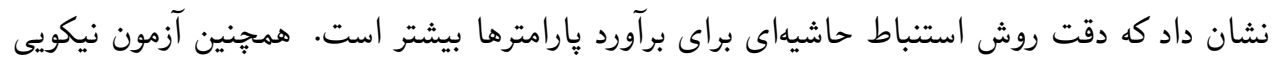

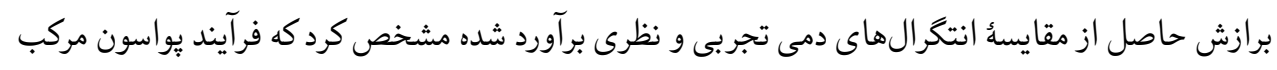

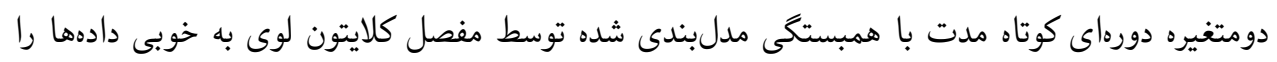



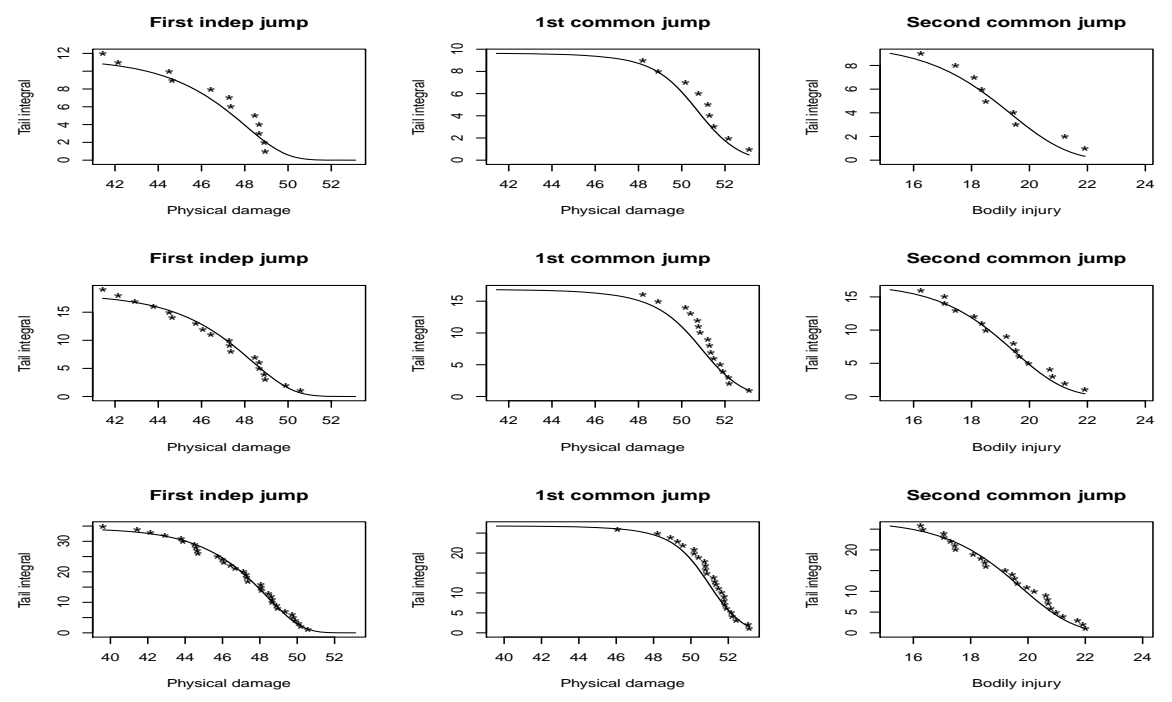

شكل ب: انتكرال دمى نظرى و تجربى متغيرهاى X و Y براى ماههاى فروردين (بالا)، ارديبهشت (وسط) و خرداد (يائين)
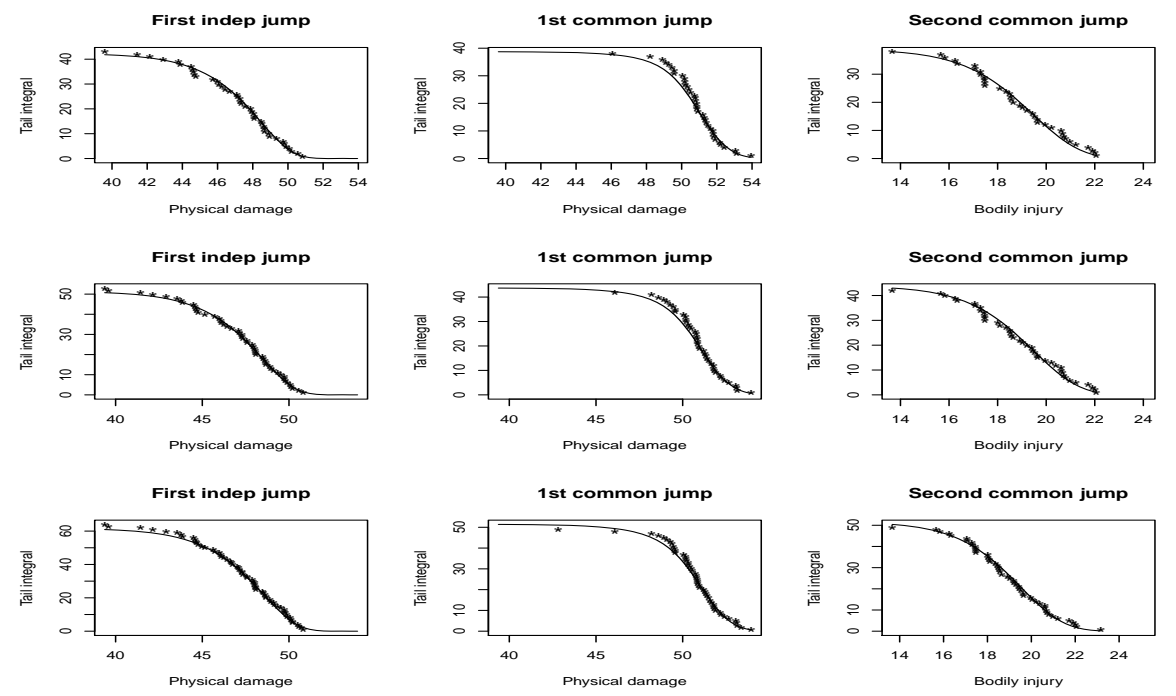

شكل ץ : انتخرال دمى نظرى و تجربى متغيرهاى X و X براى ماههاى تير (بالا)،مرداد (وسط) و شهريورماه 


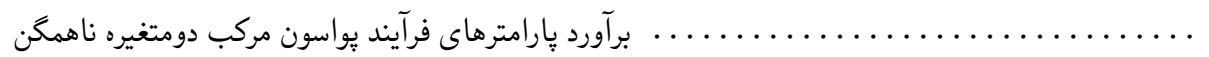
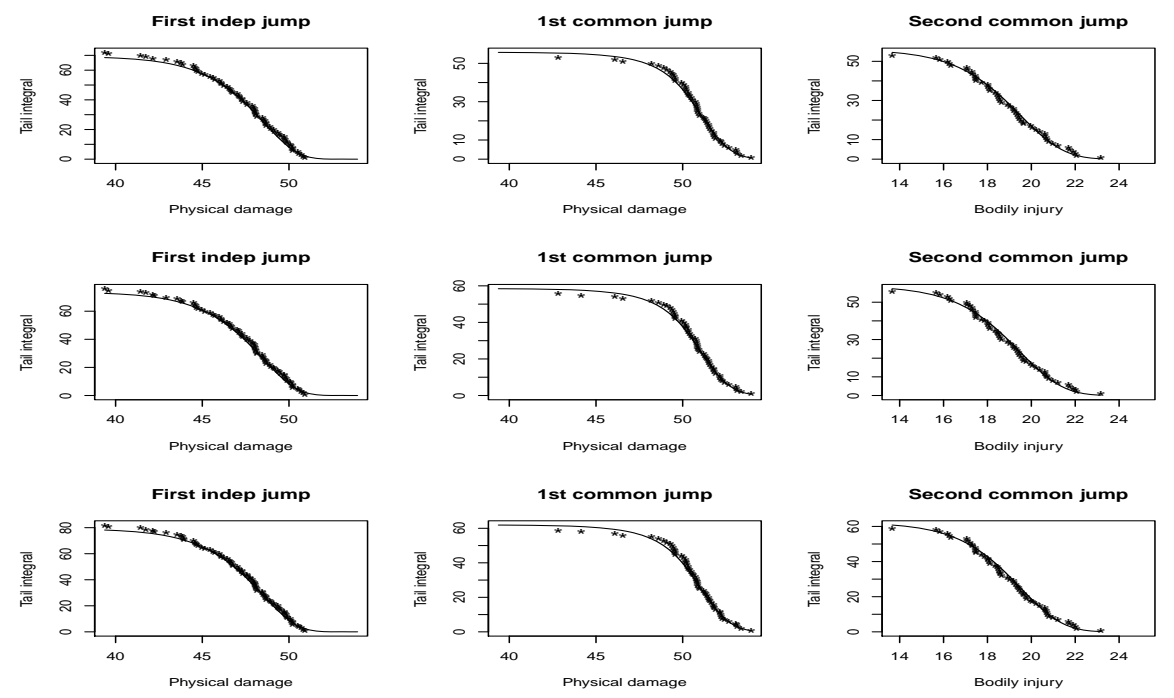

شكل ه: انتخرال دمى نظرى و تجربى متغيرهاى X و Y براى ماههاى مهر (بالا)، آبان (وسط) و آذر
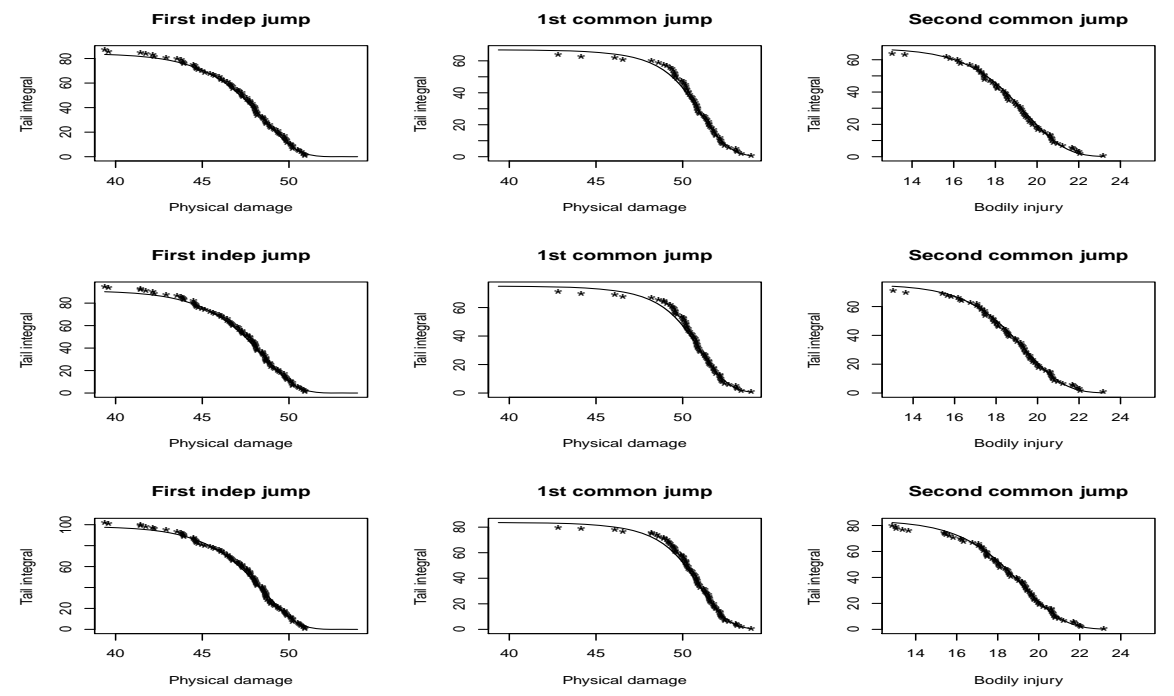

شكل 9: انتكرال دمى نظرى و تجربى متغيرهاى X و Y براى ماههاى دى (بالا)،بهمن (وسط) و اسفند 


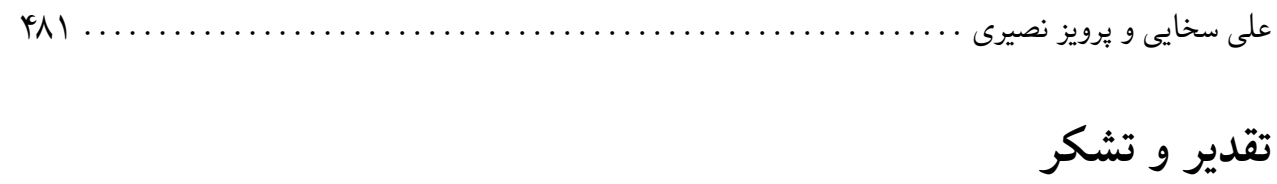

نويسندكان مقاله ضمن تشكر از سردبير محترم مجله از ييشنهادها و نظرات ارزشمند داوران و ويراستار

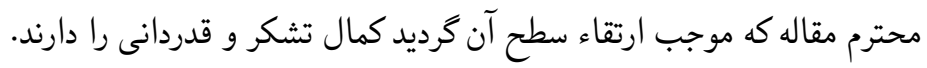

Asmussen, S. and Rolski, T. (1994), Computational Method in Risk Theory: a Matrix-Algorithmic Approach, Insurance:Mathematics and Economics, 10, 259-274.

Avanzi, B., Cassar, L. C. and Wong, B. (2011), Modelling Dependence in Insurance Claims Processes with Lévy Copulas., ASTIN Bulletin: The Journal of the IAA, 41, 575-609.

Bocker, K., and Kluppelberg, C. (2008), Modelling and Measuring Multivariate OperationalRrisk with Lévy Copulas., The Journal of Operational Risk, 3, 3-27.

Bocker, K., and Kluppelberg, C. (2009), First Order Approximations to Operational Risk-Dependence and Consequences., Operational Risk Toward Basel III, Best Practices and Issues in Modeling, Management and Regulation, Wiley, New York, 219-245.

Cont, R. and Tankov, P. (2004), Financial Modelling with Jump Processes, Chapman \& Hall/CRC, Boca Raton.

Dimitrov, B., Green Jr, D., and Chukova, S. (1997), Probability Distributions in Periodic Random Environment and Their Applications. SIAM Journal on Applied Mathematics, 57, 501-517.

Esmaeili, H., and Kluppelberg, C. (2010), Parameter Estimation of a Bivariate Compound Poisson Process. Insurance: Mathematics and Economics, 47, 224-233.

Garrido, J., and Lu, Y. (2002), On Double Periodic Non-homogeneous Poisson Processes, Universidad Carlos III, Departamento de Economia de la Empresa, Business Economics Working Papers, 2.

Joe, H. and Xu, J. J. (1996), The Estimation Method of Inference Functions for Margins for Multivariate Models. Technical Report 166, UBC, Department of Statistics. 


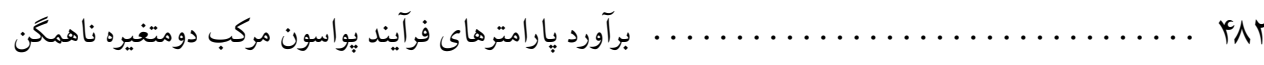

Kallsen, J., and Tankov, P. (2006), Characterization of Dependence of Multidimensional Lévy Processes Using Lévy Copulas. Journal of Multivariate Analysis, 97, 1551-1572.

Lu, Y., and Garrido, J. (2005), Doubly Periodic Non-homogeneous Poisson Models for Hurricane Data. Statistical Methodology, 2, 17-35.

Özel, G. (2011a), On Certain Properties of a Class of Bivariate Compound Poisson Distributions and an Application to Earthquake Data. Revista Colombiana de Estadistica, 34, 545-566.

Özel, G. (2011b), A New Method for Evaluation of Bivariate Compound Poisson Distribution for Aggregate Claims. Gazi University Journal of Science, 24, 241-248.

Özel, G. (2013), On the Moment Characteristics for the Univariate Compound Poisson and Bivariate Compound Poisson Processes with Applications. Revista Colombiana de Estadística, 36, 59-77.

Rolski, T., Schmidli, H., Schmidt, V. and Teugels, J. (1999), Stochastic Processes for Insurance and Finance, Wiley, New York.

Velsen, J. L. (2012), Parameter Estimation of a Lévy Copula of a Discretely Observed Bivariate Compound Poisson Process with an Application to Operational Risk Modelling. https://arxiv.org/abs/1212.0092. 
Journal of Statistical Sciences, Autumn and Winter, 2019

Vol. 13, No. 2, pp 461-482

DOI: $10.29252 /$ jss.13.2.461

\title{
Estimating the Parameters of Periodic Bivariate Compound Poisson Process by Inference for Margins Method
}

\author{
A. Sakhaei, and P. Nasiri \\ Department of Statistics, Payame Noor University, Tehran, Iran, P.O.Box \\ $19490-4997$.
}

\begin{abstract}
The non-homogeneous bivariate compound Poisson process with short term periodic intensity function is used for modeling the events with seasonal patterns or periodic trends. In this paper, this process is carefully introduced. In order to characterize the dependence structure between jumps, the Lévy copula function is provided. For estimating the parameters of the model, the inference for margins method is used. As an application, this model is fitted to an automobile insurance dataset with inference for margins method and its accuracy is compared with the full maximum likelihood method. By using the goodness of fit test, it is confirmed that this model is appropriate for describing the data.
\end{abstract}

Keywords: Inference for margins method, Lévy process, Lévy copula,Non homogeneous Poisson Process, Short-term periodic.

Mathematics Subject Classification (2010): 62H12, 60J75, 62P05 